\title{
Effect of Nitrogen Fertilizer and Fulvic Acid Application on the Growth, Productivity and Nutritional Quality of Cabbage
}

\author{
Ashraf Mahmoud ${ }^{1}$, Ali H. Abdel- Razik $^{1}$, Sanaa M. El-Araby ${ }^{1}$ and Shimaa M. Hassan ${ }^{1 *}$
}

\begin{abstract}
Two field experiments were conducted at the Agricultural Experimental Station, Alexandria University, at Abis during the two growing seasons of 2017-2018 and 2018-2019. The objective of these experiments was clarifying the response of cabbage plants to nitrogen fertilizer and soil application of fulvic acid as well as exploring its effect on the growth, yield and chemical components. Four levels of nitrogen fertilizer were tested (control treatment $0,40,60$ and $80 \mathrm{~kg} \mathrm{~N} /$ fed and three concentrations of fulvic acid $(0,100$ and $200 \mathrm{ppm}$ as a soil drench), were studied in a split plots system in a randomized complete blocks design, with three replications, in both seasons. Results indicated that raising $\mathrm{N}$ applied level from 0 up to $80 \mathrm{~kg} \mathrm{~N} /$ fed was associated with progressive increments of the unfolded leaves plant $^{-1}$ characters, marketable head characters, inner head stem characters, dry matter content $(\%)$, total yield of cabbage fed. ${ }^{-1}$ and marketable yield. Soil application of fulvic acid did not significantly reflect any effect on the tested parameters of vegetative growth characters and cabbage yield and quality characters. The obtained and discussed results of the present study showed that fertilizing cabbage plants with $\mathrm{N}$ at a rate of $80 \mathrm{~kg} \mathrm{~N} / \mathrm{fed}$ with $100 \mathrm{ppm}$ fulvic acid might be considered as an adequate and effecient treatment combination for the production of high yield of the marketable head with good quality suitable for local consumption, in the two growing seasons.
\end{abstract}

Key words: Cabbage, Nitrogen fertilizers, Fulvic acid, growth, yield.

\section{INTRODUCTION}

Cabbage (Brassica oleracea L.) is an important cole crops belonging to the family Cruciferae and the winter leafy vegetable in Egypt. It has good nutritional value of many vitamins (A, B1, B2 and C). It is rich in minerals like $\mathrm{Ca}, \mathrm{Mg}, \mathrm{P}, \mathrm{K}, \mathrm{Na}$ and $\mathrm{S}$ (Verma and Nawange, 2015). It's, also, an important source of antioxidant such as carotenoids, polyphenols and ascorbic acid (Riad et al., 2009). The area and the production of cabbage in Egypt was about 38900 fed, and 485700 ton respectively (FAO, 2018). Cabbage is a heavy feeder crop, requiring a high rate of chemical fertilizer for growth and head yield development. Heavy application of commercial organic fertilizer as a liquid humic acid eliminates the necessity for $\mathrm{N}$ side-dressings and reduces the amount of chemical fertilizer that must be applied. It could be beneficial to reduce the use of chemical fertilizers and find alternative ways to improve yield quality and quantity (Manea, 2017).

To achieve marketable cabbage with high nutritional quality, the agronomic practices which highly dependent on $\mathrm{N}$ fertilizer is required. Lack of $\mathrm{N}$ causes stunted growth or leaves discoloration in green cabbage. However, excessive applied of $\mathrm{N}$ fertilizer contributes to nitrate build up in soil and vegetables (Manchali et al., 2012). Imbalanced and poor strategy of nitrogen addition limits yields and induces large losses of reactive nitrogen to the environment; as well as could lead to increase physiological disorders of crops after harvest (Hewett, 2006). Cabbage has high nitrogen uptake rates and high $\mathrm{N}$ contents, which is required for higher yield (Riad et al., 2009; Din et al., 2007). An adequate supply of nitrogen is essential for vegetative growth, head formation and desirable yield (Yshiwas, 2017).

Fulvic acid (FA) is a mixture of weak aliphatic and aromatic organic acid as well as a part of humic substances (HS) that soluble in water at all $\mathrm{pH}$ conditions (acidic, neutral and alkaline). The fulvic acid is an important fraction of soil organic matter (VanHees et al., 2005).Several hypotheses interpretiate how humic substances may function in plants to produce changes in growth. Some possible mechanisms are direct and indirect effects (Selim and Mossa, 2012). Concerning the direct effects, it has been demonstrated that HS could induce an increase in the root surface by affecting root morphology (Schmidt et al., 2007). The growth and yield of cabbage crop are remarkably influenced by organic and inorganic nutrients .It is an established fact that use of inorganic fertilizer for the crops is not so good for health because of its residual effect, but in the case of organic fertilizer such as problem dose not arise, whearas; it increase the productivity of soil as well as crop quality and yield (Tindall, 1983 and 2000). Excess application of inorganic fertilizer causes a hazard to the environment. Unfortunately, the application of both organic and

DOI: 10.21608/asejaiqjsae.2021.206156

${ }^{1}$ Department of Vegetable, Faculty of Agriculture,

Alexandria University, 21545- El Shatby, Alexandria, Egypt.

*Corresponding author: shaymaa.hassan@alexu.edu.eg

Received October 15, 2021, Accepted, November 23, 2021. 
inorganic fertilizer combined can increase the yield as well as keep the environment sound (Hsieh et al., 1995). The current study was conducted to investigate effect of various nitrogen concentrations and fulvic acid on growth, productivity and nutritional quality characteristics of cabbage.

\section{MATERIALS AND METHODS}

\section{Soil Analysis of Experimental Sites}

Prior to the initiation of each experiment, soil samples to 20-25 cm depth from the experimental sites were collected and analyzed for some chemical and physical properties, according to the published and standard procedures described by (A.O.A.C., 1992). The values of these analyses are shown in Table (1).

Two field experiments were carried out during the autumn seasons of 2017/2018 and 2018/2019, at the Experimental Station Farm of the Faculty of Agriculture, at Abies, Alexandria University, Egypt. Main plots were assigned to the four nitrogen levels $(0$, 40,60 and $80 \mathrm{~kg} \mathrm{~N} /$ feddan), denoted as $\mathrm{N}_{0}, \mathrm{~N}_{1}, \mathrm{~N}_{2}$ and $\mathrm{N}_{3}$, respectively). The sub-plots was allocated to three concentrations of fulvic acid; 0, 100, $200 \mathrm{ppm}$, will be denoted as $F_{0}, F_{1}$, and $F_{2}$, respectively. Ammonium sulphate $(20 \% \mathrm{~N})$ and ammonium nitrate $(33.5 \% \mathrm{~N})$ were the respective source of nitrogen fertilizer. Application of $\mathrm{N}$ levels was side banded.

Fulvic acid treatments were applied as a drenching methods to each plant root area three times during the vegetative growth period at three weeks intervals. The first one was carried after four weeks fom transplanting, in both studied seasons. The volume of the tested concentrations of fulvic acid is (0.2 Liter) using a hand sprayer previously modified and calibrated.Cabbage transplants (c.v. Balady); were transplanted on October 22, 2017 and October 10, 2018 at interow spacing of 40 $\mathrm{cm}$; in the first and second seasons, respectively. A full dose of P2O5 (90 kg P2O5/fed.) as a calcium super phosphate (15\% P2O5) and $\mathrm{K} 2 \mathrm{O}(60 \mathrm{~kg} \mathrm{~K} 2 \mathrm{O} / \mathrm{fed}$.) as a potassium sulphate (48-50\% K2O) were applied during soil preparation. Regular standard agricultural practices such irrigation (surface system), cultivation, control of disease and pest were carried out as recommended by the Egyptian Ministry of Agriculture. The first hoeing and weeding were carried out 3 weeks after transplanting and two more weedings carried out at one month interval.

\section{Data Recorded.}

Vegetative growth characters; a random sample of five cabbage plants was taken throughout the harvesting in growing seasons to measure number of unfolded leaves plant ${ }^{-1}$ characters, marketable head characters, inner head stem characters and dry matter content.

Cabbage yield and quality characters; Harvesting was started after 103 and 120 days after transplanting in the two growing seasons, respectively. Total yield of cabbage fed..$^{-1}$, marketable yield, fresh weight of plant and nitrogen use efficiency (NUE)

Chemical constituents of cabbage leaves; random samples of the cabbage plants, were randomly collected from each sub-plot before planting, then washed with distilled water, weighed, then oven dried at $70{ }^{\circ} \mathrm{C}$ till constant weight. The dried leaf materials were ground and homogenized, wet digested; using concentrated sulfuric acid and $\mathrm{H}_{2} \mathrm{O}_{2}$, and the total nitrogen and phosphorus on leaves of cabbage were determined calorimetrically; using spectrophotometer at 662 and 650 nanometers; according to Evenhuis, (1976) and Murphy and Riley, (1962), respectively. The total chlorophyll of leaves $(\mathrm{mg} / 100 \mathrm{gm}$ fresh weight) was determined using the colorimetric method at wavelength of 660 and $642.5 \mathrm{~mm}$, respectively (Witham et al.,1971) and the total carbohydrate of inedible leaves was determined accordingly (A.O.A.C., 1992).

Table 1. Some physical and chemical properties of the two experimental sites used in the two-growing season.

\begin{tabular}{lcc}
\hline Properties & Season 2017/2018 & Season 2018/2019 \\
\hline Physical properties & & \\
\hline Clay (\%) & 44.8 & 42.9 \\
Silt (\%) & 20.9 & 24.6 \\
Sand (\%) & 34.3 & 32.5 \\
Soil texture & Clay loam & Clay loam \\
\hline Chemical properties & & \\
\hline PH & 7.93 & 8.12 \\
E.C (dS/m) & 3.32 & 3.41 \\
O.M. $(\%)$ & 1.05 & 1.20 \\
Total N (\%) & 1.05 & 1.11 \\
Available phosphorus, ppm & 3.18 & 2.75 \\
\hline
\end{tabular}

* These analyses were carried out at the central laboratory, Faculty of Agriculture Alexandria University. 


\section{Statistical analysis}

Statistical analysis was performed using analysis of variance (ANOVA). All obtained data of the two field experiments were statistically analyzed using Co-state software and Revised L.S.D. test at 0.05 level was used to compare the differences among the means of the various treatment combinations, as illustrated by Snedecor and Cochran (1980).

\section{RESULTS AND DISCUSSION}

\section{Vegetative growth characters}

Results of the two seasons revealed that increasing the rate of $\mathrm{N}$ fertilizer from 0 up to $80 \mathrm{~kg} / \mathrm{fed}$ was associated with constant increases. The highest mean value was recorded with $\mathrm{N}$ at $80 \mathrm{~kg} / \mathrm{fed}$ (Tables 2 - 3). However, the three $\mathrm{N}$ levels in the first season and the highest two levels (60 and $80 \mathrm{~kg} \mathrm{~N} / \mathrm{fed}$ ) in the second season were not significantly differed in their effects on the percentage of unfolded leaves to either head or plant weight. The number of inedible leaves per plant constantly and significantly increased with increments of $\mathrm{N}$ rate up to $80 \mathrm{~kg} / \mathrm{fed}$ in the first season but the differences among the three $\mathrm{N}$ levels did not reach to the level of significance, in the second season. The dry matter content of outer leaves was negatively correlated with the applied $\mathrm{N}$ rates in the first season, however, the differences among $\mathrm{N}_{1}, \mathrm{~N}_{2}$ and $\mathrm{N}_{3}$ were not significant. Meanwhile, the differences in the second season were not significant in this respect, compared with control treatment $\left(\mathrm{N}_{0}\right)$. The detective positive increments on the studied characters may be attributed to the essential roles of $\mathrm{N}$ in the development and differentiation of unfolded leaves since $\mathrm{N}$ plays a major role in protein and nucleic acids synthesis and protoplasm formation. Moreover, it stimulates the meristimic activity, which, in turn, resulted in more new tissues and organs (Russel, 1973 and Yagodin, 1984). The importance of $\mathrm{N}$ on plant growth may, also, owing to essentially of $\mathrm{N}$ as a plant nutrient as well as its importance in the metabolism of many constituents such as chlorophyll, amino acid, auxin, enzymes and general protein synthesis (Singh, 2008). They added, also, that an adequate of $\mathrm{N}$ supply generally, stimulates not only photosynthesis but also amino acids and protein. The results of the two seasons revealed that, the characters of inedible leaves of cabbage plant did not significantly respond to applications of fulvic acid, the exception was found with the percentages of outer leaves to head and plant weights in the second season, where plants treated with $\mathrm{F}_{2}(200 \mathrm{ppm})$ significantly attained the highest values (45.6 and $85.8 \%$ ), respectively compared with control treatment $\mathrm{F}_{0}(0 \mathrm{ppm})$.

Table 2. Unfolded leaves characters of cabbage plant as affected by nitrogen fertilizer rates and fulvic acid concentrations in the winter seasons of 2017/2018 and 2018/2019.

\begin{tabular}{|c|c|c|c|c|c|c|}
\hline \multicolumn{2}{|c|}{ Treatments } & \multicolumn{5}{|c|}{ Unfolded leaves plant $^{-1}$} \\
\hline $\begin{array}{l}\text { Nitrogen fertilizer rates } \\
\left(\mathrm{kg} \mathrm{fed}^{-1}\right)\end{array}$ & $\begin{array}{l}\text { Fulvic acid } \\
\quad(\text { ppm) }\end{array}$ & Number & $\begin{array}{c}\text { Fresh } \\
\text { Wt. } \\
(\text { g) } \\
\end{array}$ & $\begin{array}{c}\% \\
\text { plant } \\
\text { Wt. }\end{array}$ & $\begin{array}{c}\% \\
\text { head } \\
\text { Wt. }\end{array}$ & $\begin{array}{c}\text { DM } \\
\text { content } \\
(\%)\end{array}$ \\
\hline & & Winter & $2017 / 2018$ & & & \\
\hline $0\left(\mathrm{~N}_{0}\right)$ & & $10.6 \mathrm{c}$ & $368 \mathrm{~b}$ & $54.1 \mathrm{a}$ & $120.2 \mathrm{a}$ & $19.7 \mathrm{a}$ \\
\hline $40\left(\mathrm{~N}_{1}\right)$ & & $12.3 \mathrm{~b}$ & $1319 \mathrm{a}$ & $43.7 \mathrm{~b}$ & $73.2 \mathrm{~b}$ & $15.4 \mathrm{~b}$ \\
\hline $60\left(\mathrm{~N}_{2}\right)$ & & $13.0 \mathrm{ab}$ & $1366 \mathrm{a}$ & $44.3 \mathrm{~b}$ & $79.6 \mathrm{~b}$ & $12.7 \mathrm{~b}$ \\
\hline \multirow[t]{5}{*}{$80\left(\mathrm{~N}_{3}\right)$} & & $14.1 \mathrm{a}$ & $1543 \mathrm{a}$ & $43.1 \mathrm{~b}$ & $78.2 \mathrm{~b}$ & $13.3 \mathrm{~b}$ \\
\hline & $0\left(\mathrm{~F}_{0}\right)$ & $12.7 \mathrm{a}$ & $1148 \mathrm{a}$ & $46.8 \mathrm{a}$ & $86.9 \mathrm{a}$ & $14.4 \mathrm{a}$ \\
\hline & $100\left(\mathrm{~F}_{1}\right)$ & $12.2 \mathrm{a}$ & $1156 \mathrm{a}$ & $47.3 \mathrm{a}$ & $91.4 \mathrm{a}$ & $16.4 \mathrm{a}$ \\
\hline & $200\left(\mathrm{~F}_{2}\right)$ & $12.6 \mathrm{a}$ & $1142 \mathrm{a}$ & $45.4 \mathrm{a}$ & $85.2 \mathrm{a}$ & $15.0 \mathrm{a}$ \\
\hline & & Winter & 2018 / 2019 & & & \\
\hline $0\left(\mathrm{~N}_{0}\right)$ & & $13.6 \mathrm{a}$ & $1665 \mathrm{~b}$ & $49.5 \mathrm{a}$ & $99.3 \mathrm{a}$ & $12.2 \mathrm{a}$ \\
\hline $40\left(\mathrm{~N}_{1}\right)$ & & $12.0 \mathrm{a}$ & $1929 \mathrm{a}$ & $45.1 \mathrm{~b}$ & $83.7 \mathrm{~b}$ & $11.9 \mathrm{a}$ \\
\hline $60\left(\mathrm{~N}_{2}\right)$ & & $11.1 \mathrm{a}$ & 1949 a & $41.1 \mathrm{c}$ & $70.9 \mathrm{c}$ & $10.5 \mathrm{a}$ \\
\hline \multirow[t]{4}{*}{$80\left(\mathrm{~N}_{3}\right)$} & & $12.7 \mathrm{a}$ & $2068 \mathrm{a}$ & $41.6 \mathrm{c}$ & $72.6 \mathrm{c}$ & $10.9 \mathrm{a}$ \\
\hline & $0\left(\mathrm{~F}_{0}\right)$ & $12.3 \mathrm{a}$ & $1923 \mathrm{a}$ & $43.1 \mathrm{~b}$ & $77.9 \mathrm{~b}$ & $11.6 \mathrm{a}$ \\
\hline & $100\left(\mathrm{~F}_{1}\right)$ & $12.8 \mathrm{a}$ & $1866 \mathrm{a}$ & $44.3 \mathrm{ab}$ & $81.1 \mathrm{ab}$ & $11.5 \mathrm{a}$ \\
\hline & $200\left(\mathrm{~F}_{2}\right)$ & $12.7 \mathrm{a}$ & $1919 \mathrm{a}$ & $45.6 \mathrm{a}$ & $85.8 \mathrm{a}$ & $11.2 \mathrm{a}$ \\
\hline
\end{tabular}

*values marked with alphabetical letter (s), within a comparable group of means, do not significantly differ, using revised L.S.D test at 0.05 level. 
Table 3. Unfolded leaves characters of cabbage plant as affected by the interaction between nitrogen fertilizer rates and fulvic acid concentrations in the winter seasons of 2017/2018 and 2018/2019.

\begin{tabular}{|c|c|c|c|c|c|c|}
\hline \multicolumn{2}{|c|}{ Treatments } & \multicolumn{5}{|c|}{ Unfolded leaves plant ${ }^{-1}$} \\
\hline $\begin{array}{l}\text { Nitrogen } \\
\text { fertilizer } \\
\left(\mathrm{kg} \mathrm{fed}^{-1}\right)\end{array}$ & $\begin{array}{l}\text { Fulvic acid } \\
\quad(\mathbf{p p m})\end{array}$ & Number & $\begin{array}{c}\text { Fresh } \\
\text { Wt. } \\
(\mathrm{g})\end{array}$ & $\begin{array}{c}\% \\
\text { plant } \\
\text { Wt. }\end{array}$ & $\begin{array}{c}\% \\
\text { head } \\
\text { Wt. }\end{array}$ & $\begin{array}{c}\text { DM } \\
\text { content } \\
(\%)\end{array}$ \\
\hline \multicolumn{7}{|c|}{ Winter $2017 / 2018$} \\
\hline \multirow{4}{*}{$0\left(\mathrm{~N}_{0}\right)$} & $0\left(\mathrm{~F}_{0}\right)$ & $9.9 \mathrm{~d}$ & $308 \mathrm{e}$ & $52.6 \mathrm{a}$ & $113.1 \mathrm{~b}$ & $19.5 \mathrm{a}$ \\
\hline & $100\left(\mathrm{~F}_{1}\right)$ & $10.9 \mathrm{~d}$ & $407 \mathrm{e}$ & $57.4 \mathrm{a}$ & $137.9 \mathrm{a}$ & $19.4 \mathrm{a}$ \\
\hline & $200\left(\mathrm{~F}_{2}\right)$ & $10.6 \mathrm{~d}$ & $388 \mathrm{e}$ & $52.3 \mathrm{a}$ & $109.5 \mathrm{~b}$ & $20.2 \mathrm{a}$ \\
\hline & $0\left(\mathrm{~F}_{0}\right)$ & $12.8 \mathrm{bc}$ & $1379 \mathrm{bcd}$ & $44.9 \mathrm{~b}$ & $77.8 \mathrm{c}$ & $13.7 \mathrm{c}$ \\
\hline \multirow[t]{3}{*}{$40\left(\mathrm{~N}_{1}\right)$} & $100\left(\mathrm{~F}_{1}\right)$ & $11.1 \mathrm{~cd}$ & $1245 \mathrm{~d}$ & $44.5 \mathrm{~b}$ & $68.7 \mathrm{c}$ & $18.6 \mathrm{ab}$ \\
\hline & $200\left(\mathrm{~F}_{2}\right)$ & $13.1 \mathrm{~b}$ & $1334 \mathrm{~cd}$ & $41.7 \mathrm{~b}$ & $73.2 \mathrm{c}$ & $13.7 \mathrm{c}$ \\
\hline & $0\left(\mathrm{~F}_{0}\right)$ & $12.9 \mathrm{~b}$ & $1344 \mathrm{~cd}$ & $44.6 \mathrm{~b}$ & $78.6 \mathrm{c}$ & $12.3 \mathrm{c}$ \\
\hline \multirow[t]{3}{*}{$60\left(\mathrm{~N}_{2}\right)$} & $100\left(\mathrm{~F}_{1}\right)$ & $13.0 \mathrm{~b}$ & $1348 \mathrm{~cd}$ & $44.6 \mathrm{~b}$ & $83.4 \mathrm{c}$ & $12.7 \mathrm{c}$ \\
\hline & $200\left(\mathrm{~F}_{2}\right)$ & $13.2 \mathrm{~b}$ & 1407 bcd & $43.6 \mathrm{~b}$ & $76.8 \mathrm{c}$ & $13.2 \mathrm{c}$ \\
\hline & $0\left(\mathrm{~F}_{0}\right)$ & $15.1 \mathrm{a}$ & $1563 \mathrm{ab}$ & $44.9 \mathrm{~b}$ & $77.9 \mathrm{c}$ & $12.1 \mathrm{c}$ \\
\hline \multirow[t]{4}{*}{$80\left(\mathrm{~N}_{3}\right)$} & $100\left(\mathrm{~F}_{1}\right)$ & $13.9 \mathrm{ab}$ & $1626 \mathrm{a}$ & $42.9 \mathrm{~b}$ & $75.6 \mathrm{c}$ & $14.7 \mathrm{bc}$ \\
\hline & \multirow{2}{*}{\multicolumn{6}{|c|}{$\begin{array}{l}13.3 \mathrm{~b} \\
\text { Winter } 2018 / 2019\end{array}$}} \\
\hline & & & & & & \\
\hline & $0\left(\mathrm{~F}_{0}\right)$ & $13.1 \mathrm{abc}$ & $1707 \mathrm{~cd}$ & $48.8 \mathrm{ab}$ & $97.2 \mathrm{ab}$ & $12.2 \mathrm{a}$ \\
\hline \multirow[t]{3}{*}{$0\left(\mathrm{~N}_{0}\right)$} & $100\left(\mathrm{~F}_{1}\right)$ & $14.2 \mathrm{a}$ & $1643 \mathrm{~d}$ & $48.2 \mathrm{ab}$ & $93.4 \mathrm{~b}$ & $12.8 \mathrm{a}$ \\
\hline & $200\left(F_{2}\right)$ & $13.4 \mathrm{ab}$ & $1644 \mathrm{~d}$ & $51.7 \mathrm{a}$ & $107.4 \mathrm{a}$ & $11.7 \mathrm{a}$ \\
\hline & $0\left(\mathrm{~F}_{0}\right)$ & $11.6 \mathrm{~cd}$ & $1870 \mathrm{bcd}$ & $42.0 \mathrm{de}$ & $73.4 \mathrm{~d}$ & $12.5 \mathrm{a}$ \\
\hline \multirow[t]{3}{*}{$40\left(\mathrm{~N}_{1}\right)$} & $100\left(\mathrm{~F}_{1}\right)$ & $11.9 \mathrm{bcd}$ & $1888 \mathrm{bc}$ & $45.9 \mathrm{bc}$ & $86.4 \mathrm{bc}$ & $11.4 \mathrm{a}$ \\
\hline & $200\left(\mathrm{~F}_{2}\right)$ & $12.6 \mathrm{a}-\mathrm{d}$ & $2029 a b$ & $47.6 \mathrm{~b}$ & $91.1 \mathrm{~b}$ & $11.8 \mathrm{a}$ \\
\hline & $0\left(\mathrm{~F}_{0}\right)$ & $11.0 \mathrm{~d}$ & $1881 \mathrm{bc}$ & $38.8 \mathrm{e}$ & $64.9 \mathrm{~d}$ & $10.8 \mathrm{a}$ \\
\hline \multirow[t]{3}{*}{$60\left(\mathrm{~N}_{2}\right)$} & $100\left(\mathrm{~F}_{1}\right)$ & $12.5 \mathrm{a}-\mathrm{d}$ & 1929 bc & $41.4 \mathrm{de}$ & $71.2 \mathrm{~d}$ & $10.5 \mathrm{a}$ \\
\hline & $200\left(F_{2}\right)$ & $12.5 \mathrm{a}-\mathrm{d}$ & $2037 \mathrm{ab}$ & $42.9 \mathrm{~cd}$ & $76.6 \mathrm{~cd}$ & $10.3 \mathrm{a}$ \\
\hline & $0\left(\mathrm{~F}_{0}\right)$ & $13.4 \mathrm{abc}$ & $2233 \mathrm{a}$ & $42.8 \mathrm{~cd}$ & $76.2 \mathrm{~cd}$ & $10.8 \mathrm{a}$ \\
\hline \multirow[t]{2}{*}{$80\left(\mathrm{~N}_{3}\right)$} & $100\left(\mathrm{~F}_{1}\right)$ & $12.4 \mathrm{a}-\mathrm{d}$ & $2003 \mathrm{ab}$ & $41.9 \mathrm{de}$ & $73.4 \mathrm{~d}$ & $11.2 \mathrm{a}$ \\
\hline & $200\left(\mathrm{~F}_{2}\right)$ & $12.3 \mathrm{bcd}$ & $1966 \mathrm{~b}$ & $40.1 \mathrm{de}$ & $68.2 \mathrm{~d}$ & $10.9 \mathrm{a}$ \\
\hline
\end{tabular}

*values marked with alphabetical letter (s), within a comparable group of means, do not significantly differ, using revised L.S.D test at 0.05 level.

The detective insignificant effects of the applied fulvic acid on most of the studied characters of the above ground plant organs and the analyzed chemical constituents may be attributed to the relatively high content of the native soil dissolved organic matter (DOM) in the soils of the experimental sites; 1.05 and $1.20 \%$ as shown in Table (1), in the first and second seasons, respectively, which disappeared the improving effects of the applied rates of fulvic acid. This interpretation is in accordance with those of Hartz and Bottoms (2010), who reported that, at typical commercial application rate in representative field soils, humic substances (HS) is unlikely to be significantly improved vegetable crop nutrient uptake or productivity. The vast majority of positive reports have come from hydroponic or sand culture experiments (Chen et al., 2004). US research suggests that under representative field conditions, commercial HA formulations do not reliably provide agronomic benefits for vegetable production. Boyhan et al. (2001) found that HS had no effects on onion yield in 3 years of field trials. Similarly Feibert et al. (2003) and Duval et al. (1998) reflect no benefit from HS application in field production of onions and mustard greens, respectively. Concerning the comparisons among the means of various treatment combinations of $\mathrm{N}$ fertilizer and fulvic acid levels (Table 3), the highest mean values were obtained from the unfertilized plots with $\mathrm{N}$ and treated with fulvic acid at rate of $F_{1}$ or $F_{2}(100$ or 200 ppm).

The results presented in Tables (4- 5) clarify the effects of $\mathrm{N}$ fertilization, fulvic acid and their interaction on marketable head characters in the two winter seasons. 
The three tested $\mathrm{N}$ levels; $\mathrm{N}_{1}, \mathrm{~N}_{2}$ and $\mathrm{N}_{3}$ (40, 60 and 80 $\mathrm{kg} / \mathrm{fed}$ ) did not significantly differed in their effects on the studied parameters of the marketable head in the two growing seasons. As for the influences of the studied $\mathrm{N}$ levels on the compactness of marketable head, the results of the first season showed that fertilizing cabbage plant with $\mathrm{N}_{1}, \mathrm{~N}_{2}$ and $\mathrm{N}_{3}(40,60$ or $80 \mathrm{~kg}$ $\mathrm{N} /$ fed) ,statistically, produced heads with the highest compactness as compared with those of the unfertilized treatment. The highest compactness was attained with $\mathrm{N} 3$ (80 kg N/fed). However, the results of the second season indicated that the three $\mathrm{N}$ levels did not affect head compactness as compared with the control treatment $\left(\mathrm{N}_{0}\right)$. The results of the present study are agreed to a great extent with those reported by Riad et al. (2009) and Haque et al. (2015), who illustrated that fresh weight, length and diameter of cabbage head were associated with increasing $\mathrm{N}$ levels up to 90 and $350 \mathrm{~kg}$ N/ha, respectively. Recently, Yshiwas (2017) revealed that the highest length, diameter and weight of cabbage head were obtained from $150 \mathrm{~kg} \mathrm{~N} / \mathrm{ha}$, while the lowest dry weight was attained at $0 \mathrm{Kg} \mathrm{N} / \mathrm{ha}$. These findings are also in accordance with those of Patrick et al. (2012), who indicated that head diameter increased from $98 \mathrm{~mm}$ to $218 \mathrm{~mm}$ with increasing $\mathrm{N}$ level from 0 to 120 $\mathrm{kg} / \mathrm{ha}$. Concerning the results of the second season , results indicated that application of fulvic acid at rates $F_{1}$ and $F_{2}$,significantly, decreased head fresh weight, head weight percentage to plant weight, number and weight of edible head leaves. Meanwhile, head length, diameter and compactness were not significantly responded to addition of fulvic acid either at $F_{1}$, or $F_{2}$. Concerning the effect of the present interaction between $\mathrm{N}$ fertilization rate and fulvic acid Table (5), the highest mean values of fresh weights of head and edible leaves, were recorded with plants fertilized with $\mathrm{N}_{2}$ or $\mathrm{N}_{3}$ combined with any level of applied fulvic acid. On the other hand, the treatment combinations of the three $\mathrm{N}$ levels; $\mathrm{N}_{1}, \mathrm{~N}_{2}$ and $\mathrm{N}_{3}$ combined with the three levels of $\mathrm{FA} ; \mathrm{F}_{0}, \mathrm{~F}_{1}$ and $\mathrm{F}_{2}$, were not significantly differed in their effects on the studied head characters but exceeded those of unfertilized with $\mathrm{N}$ fertilizer combined with any tested level of FA.

The results illustrated the effects of $\mathrm{N}$ fertilizer rates fulvic acid concentrations and their interactions, on the tested parameters of inner head stem; fresh weight, length and diameter of stem as well as the percentage of stem to head weight and its dry matter content, in the two growing seasons are presented in Tables $(6-7)$. Generally, the three $\mathrm{N}$ levels; $\mathrm{N}_{1}, \mathrm{~N}_{2}$ and $\mathrm{N}_{3}$, ,significantly, increased fresh weight, length, and diameter of inner head stem compared with control treatment $\left(\mathrm{N}_{0}\right)$. However, increasing $\mathrm{N}$ rate up to $\mathrm{N}_{3}(80$ $\mathrm{kg} \mathrm{N} / \mathrm{fed}$.) was associated with progressive and significant decreases in the percentages of the stem to head weight, in the two seasons, and its dry matter content, in the first season, compared with those of unfertilized control $\left(\mathrm{N}_{0}\right)$.

Table 4. Marketable head characters of cabbage as affected by nitrogen fertilizer rates and fulvic acid concentrations in the winter seasons of 2017/2018 and 2018/2019.

\begin{tabular}{|c|c|c|c|c|c|c|}
\hline \multicolumn{2}{|c|}{ Treatments } & \multicolumn{5}{|c|}{ Marketable head characters } \\
\hline $\begin{array}{c}\text { Nitrogen fertilizer rates } \\
\left(\mathrm{kg} \mathrm{fed}^{-1}\right)\end{array}$ & $\begin{array}{c}\text { Fulvic acid } \\
\text { (ppm) }\end{array}$ & $\begin{array}{l}\text { Fresh } \\
\text { Wt.(g) }\end{array}$ & $\begin{array}{c}\text { Length } \\
\text { (cm) }\end{array}$ & $\begin{array}{l}\text { Diameter } \\
(\mathrm{cm} .)\end{array}$ & $\begin{array}{c}\text { \% plant } \\
\text { Wt. }\end{array}$ & $\begin{array}{l}\text { Compact-ness } \\
\left(\mathrm{cm}^{3} \mathrm{~g}^{-1}\right)\end{array}$ \\
\hline \multicolumn{7}{|c|}{ Winter $2017 / 2018$} \\
\hline $0\left(\mathrm{~N}_{0}\right)$ & & $311 \mathrm{~b}$ & $14.1 \mathrm{~b}$ & $13.3 \mathrm{~b}$ & $45.9 \mathrm{~b}$ & $2.46 \mathrm{a}$ \\
\hline $40\left(\mathrm{~N}_{1}\right)$ & & $1717 \mathrm{a}$ & $19.9 \mathrm{a}$ & $21.2 \mathrm{a}$ & $56.3 \mathrm{a}$ & $1.68 \mathrm{~b}$ \\
\hline $60\left(\mathrm{~N}_{2}\right)$ & & $1805 \mathrm{a}$ & $19.7 \mathrm{a}$ & $21.4 \mathrm{a}$ & $56.9 \mathrm{a}$ & $1.70 \mathrm{~b}$ \\
\hline $80\left(\mathrm{~N}_{3}\right)$ & & $2042 \mathrm{a}$ & $18.9 \mathrm{a}$ & $21.5 \mathrm{a}$ & $57.4 \mathrm{a}$ & $1.54 \mathrm{~b}$ \\
\hline & $0\left(\mathrm{~F}_{0}\right)$ & $1447 \mathrm{a}$ & $18.2 \mathrm{a}$ & $19.4 \mathrm{a}$ & $55.1 \mathrm{a}$ & $1.89 \mathrm{a}$ \\
\hline & $100\left(\mathrm{~F}_{1}\right)$ & $1440 \mathrm{a}$ & $18.4 \mathrm{a}$ & $19.2 \mathrm{a}$ & $52.7 \mathrm{a}$ & $1.88 \mathrm{a}$ \\
\hline & $200\left(\mathrm{~F}_{2}\right)$ & $1520 \mathrm{a}$ & $17.9 \mathrm{a}$ & $19.4 \mathrm{a}$ & $546 a$ & $1.78 \mathrm{a}$ \\
\hline \multicolumn{7}{|c|}{ Winter $\quad 2018 / 2019$} \\
\hline $0\left(\mathrm{~N}_{0}\right)$ & & $1694 \mathrm{c}$ & $22.4 \mathrm{~b}$ & $22.7 \mathrm{~b}$ & $504 \mathrm{c}$ & $2.10 \mathrm{a}$ \\
\hline $40\left(\mathrm{~N}_{1}\right)$ & & $2341 \mathrm{~b}$ & $24.4 \mathrm{ab}$ & $25.1 \mathrm{a}$ & $54.8 \mathrm{~b}$ & $2.06 \mathrm{a}$ \\
\hline $60\left(\mathrm{~N}_{2}\right)$ & & $2851 \mathrm{a}$ & $26.2 \mathrm{a}$ & $25.2 \mathrm{a}$ & $58.9 \mathrm{a}$ & $1.71 \mathrm{a}$ \\
\hline \multirow[t]{4}{*}{$80\left(\mathrm{~N}_{3}\right)$} & & $2911 \mathrm{a}$ & $25.9 \mathrm{a}$ & $24.9 \mathrm{a}$ & $58.0 \mathrm{ab}$ & $1.61 \mathrm{a}$ \\
\hline & $0\left(\mathrm{~F}_{0}\right)$ & $2603 a$ & $25.3 \mathrm{a}$ & $24.8 \mathrm{a}$ & $56.9 \mathrm{a}$ & $1.83 \mathrm{a}$ \\
\hline & $100\left(\mathrm{~F}_{1}\right)$ & $2369 \mathrm{~b}$ & $24.6 \mathrm{a}$ & $24.4 \mathrm{a}$ & $55.6 \mathrm{ab}$ & $1.91 \mathrm{a}$ \\
\hline & $200\left(\mathrm{~F}_{2}\right)$ & $2377 \mathrm{~b}$ & $24.3 \mathrm{a}$ & $24.2 \mathrm{a}$ & $54.1 \mathrm{~b}$ & $1.88 \mathrm{a}$ \\
\hline
\end{tabular}

*values marked with alphabetical letter (s), within a comparable group of means, do not significantly differ, using revised L.S.D test at 0.05 level. 
Table 5. Marketable head characters of cabbage as affected by the interaction between nitrogen fertilizer rates and fulvic acid concentrations in the winter seasons of 2017/2018 and 2018/2019.

\begin{tabular}{|c|c|c|c|c|c|c|}
\hline \multicolumn{2}{|c|}{ Treatments } & \multicolumn{5}{|c|}{ Marketable head characters } \\
\hline $\begin{array}{l}\text { Nitrogen fertilizer } \\
\quad\left(\mathrm{kg} \mathrm{fed}^{-1}\right)\end{array}$ & $\begin{array}{l}\text { Fulvic acid } \\
\text { (ppm) }\end{array}$ & $\begin{array}{r}\text { Fresh } \\
\text { Wt. }(g)\end{array}$ & $\begin{array}{l}\text { Length } \\
\text { (cm) }\end{array}$ & $\begin{array}{l}\text { Diameter } \\
\text { (cm.) }\end{array}$ & $\begin{array}{l}\text { \% Plant } \\
\text { Wt. }\end{array}$ & $\begin{array}{c}\text { Compactness } \\
\left(\mathrm{cm}^{3} \mathrm{~g}^{-1}\right)\end{array}$ \\
\hline \multicolumn{7}{|c|}{ Winter $2017 / 2018$} \\
\hline \multirow{4}{*}{$0\left(\mathrm{~N}_{0}\right)$} & $0\left(\mathrm{~F}_{0}\right)$ & $266 \mathrm{e}$ & $13.7 \mathrm{c}$ & $12.5 \mathrm{~d}$ & $47.3 b$ & $2.48 \mathrm{a}$ \\
\hline & $100\left(\mathrm{~F}_{1}\right)$ & $299 \mathrm{e}$ & $14.0 \mathrm{c}$ & $13.7 \mathrm{~d}$ & $42.6 \mathrm{~b}$ & $2.71 \mathrm{a}$ \\
\hline & $200\left(\mathrm{~F}_{2}\right)$ & $369 \mathrm{e}$ & $14.7 \mathrm{c}$ & $13.6 \mathrm{~d}$ & $47.7 \mathrm{~b}$ & $2.20 \mathrm{ab}$ \\
\hline & $0\left(\mathrm{~F}_{0}\right)$ & $1681 \mathrm{~cd}$ & $20.6 \mathrm{a}$ & $21.7 \mathrm{ab}$ & $55.1 \mathrm{a}$ & $1.81 \mathrm{bc}$ \\
\hline \multirow{3}{*}{$40\left(\mathrm{~N}_{1}\right)$} & $100\left(\mathrm{~F}_{1}\right)$ & $1555 \mathrm{~d}$ & $20.0 \mathrm{a}$ & $20.1 \mathrm{c}$ & $55.5 \mathrm{a}$ & $1.58 \mathrm{c}$ \\
\hline & $200\left(\mathrm{~F}_{2}\right)$ & $1917 \mathrm{abc}$ & $19.0 \mathrm{ab}$ & $21.9 \mathrm{ab}$ & $58.3 \mathrm{a}$ & $1.64 \mathrm{c}$ \\
\hline & $0\left(\mathrm{~F}_{0}\right)$ & $1802 \mathrm{bcd}$ & $19.7 \mathrm{ab}$ & $21.3 \mathrm{abc}$ & $58.7 \mathrm{a}$ & $1.70 \mathrm{bc}$ \\
\hline \multirow[t]{2}{*}{$60\left(\mathrm{~N}_{2}\right)$} & $100\left(\mathrm{~F}_{1}\right)$ & 1744 bcd & $19.6 \mathrm{ab}$ & $21.1 \mathrm{abc}$ & $55.4 \mathrm{a}$ & $1.71 \mathrm{bc}$ \\
\hline & $200\left(\mathrm{~F}_{2}\right)$ & 1870 a-d & $19.7 \mathrm{ab}$ & $21.7 \mathrm{ab}$ & $56.4 \mathrm{a}$ & $1.69 \mathrm{bc}$ \\
\hline \multirow{3}{*}{$80\left(\mathrm{~N}_{3}\right)$} & $0\left(\mathrm{~F}_{0}\right)$ & $2038 \mathrm{ab}$ & $18.9 \mathrm{ab}$ & $22.0 \mathrm{a}$ & $59.3 \mathrm{a}$ & $1.57 \mathrm{c}$ \\
\hline & $100\left(\mathrm{~F}_{1}\right)$ & $2161 \mathrm{a}$ & $19.8 \mathrm{ab}$ & $21.9 \mathrm{ab}$ & $57.1 \mathrm{a}$ & $1.48 \mathrm{c}$ \\
\hline & $200\left(\mathrm{~F}_{2}\right)$ & $1926 \mathrm{abc}$ & $18.2 \mathrm{~b}$ & $20.5 \mathrm{bc}$ & $55.9 \mathrm{a}$ & $1.56 \mathrm{c}$ \\
\hline \multicolumn{7}{|c|}{ Winter 2018 / 2019} \\
\hline \multirow{4}{*}{$0\left(\mathrm{~N}_{0}\right)$} & $0\left(\mathrm{~F}_{0}\right)$ & $1781 \mathrm{~d}$ & $23.6 \mathrm{cde}$ & $23.5 \mathrm{bcd}$ & $51.2 \mathrm{de}$ & $2.2 \mathrm{a}$ \\
\hline & $100\left(\mathrm{~F}_{1}\right)$ & $1766 \mathrm{~d}$ & $22.3 \mathrm{de}$ & $22.2 \mathrm{~d}$ & $51.8 \mathrm{de}$ & $1.9 \mathrm{abc}$ \\
\hline & $200\left(\mathrm{~F}_{2}\right)$ & $1536 \mathrm{~d}$ & $21.2 \mathrm{e}$ & $22.5 \mathrm{~cd}$ & $48.3 \mathrm{e}$ & $2.1 \mathrm{ab}$ \\
\hline & $0\left(\mathrm{~F}_{0}\right)$ & $2577 \mathrm{~b}$ & $25.6 \mathrm{abc}$ & $24.9 \mathrm{ab}$ & $57.9 \mathrm{abc}$ & $1.9 \mathrm{abc}$ \\
\hline \multirow[t]{2}{*}{$40\left(\mathrm{~N}_{1}\right)$} & $100\left(\mathrm{~F}_{1}\right)$ & $2210 \mathrm{c}$ & $23.6 \mathrm{cde}$ & $25.7 \mathrm{a}$ & $54.1 \mathrm{~cd}$ & $2.3 \mathrm{a}$ \\
\hline & $200\left(\mathrm{~F}_{2}\right)$ & $2236 \mathrm{c}$ & $23.9 \mathrm{bcd}$ & $24.5 \mathrm{abc}$ & $52.4 \mathrm{~d}$ & $1.9 \mathrm{abc}$ \\
\hline \multirow{3}{*}{$60\left(\mathrm{~N}_{2}\right)$} & $0\left(\mathrm{~F}_{0}\right)$ & $3051 \mathrm{a}$ & $26.4 \mathrm{ab}$ & $25.5 \mathrm{ab}$ & $61.1 \mathrm{a}$ & $1.6 \mathrm{c}$ \\
\hline & $100\left(\mathrm{~F}_{1}\right)$ & $2747 \mathrm{ab}$ & $26.5 \mathrm{a}$ & $25.2 \mathrm{ab}$ & $58.5 \mathrm{ab}$ & $1.8 \mathrm{bc}$ \\
\hline & $200\left(\mathrm{~F}_{2}\right)$ & $2755 \mathrm{ab}$ & $25.6 \mathrm{abc}$ & $24.9 \mathrm{ab}$ & $57.1 \mathrm{bc}$ & $1.8 \mathrm{bc}$ \\
\hline \multirow{3}{*}{$80\left(\mathrm{~N}_{3}\right)$} & $0\left(\mathrm{~F}_{0}\right)$ & $3003 \mathrm{a}$ & $25.4 \mathrm{abc}$ & $25.4 \mathrm{ab}$ & $57.2 \mathrm{bc}$ & $1.6 \mathrm{c}$ \\
\hline & $100\left(\mathrm{~F}_{1}\right)$ & $2751 \mathrm{ab}$ & $26.0 \mathrm{abc}$ & $24.6 \mathrm{abc}$ & $58.1 \mathrm{ab}$ & $1.6 \mathrm{c}$ \\
\hline & $200\left(F_{2}\right)$ & $2981 \mathrm{a}$ & $26.6 \mathrm{a}$ & $24.9 \mathrm{ab}$ & $58.9 \mathrm{ab}$ & $1.6 \mathrm{c}$ \\
\hline
\end{tabular}

*values marked with alphabetical letter (s), within a comparable group of means, do not significantly differ, using revised L.S.D test at 0.05 level.

Table 6. Inner head stem characters of cabbage as affected by nitrogen fertilizer rates and fulvic acid concentrations in the winter seasons of $2017 / 2018$ and $2018 / 2019$.

\begin{tabular}{|c|c|c|c|c|c|c|}
\hline \multicolumn{2}{|l|}{ Treatments } & \multicolumn{5}{|c|}{ Inner head stem characters } \\
\hline $\begin{array}{l}\text { Nitrogen fertilizer rates } \\
\left(\mathrm{kg} \mathrm{fed}^{-1}\right)\end{array}$ & $\begin{array}{l}\text { Fulvic acid } \\
\quad(p p m)\end{array}$ & $\begin{array}{l}\text { Fresh Wt. } \\
\text { (g) }\end{array}$ & $\begin{array}{l}\text { Length } \\
\text { (cm) }\end{array}$ & $\begin{array}{l}\text { Diameter } \\
(\mathbf{c m})\end{array}$ & $\begin{array}{c}\text { \% Head } \\
\text { Wt. }\end{array}$ & $\begin{array}{l}\text { DM content } \\
(\%)\end{array}$ \\
\hline \multicolumn{7}{|c|}{ Winter $2017 / 2018$} \\
\hline $0\left(\mathrm{~N}_{0}\right)$ & & $74.0 \mathrm{~b}$ & $3.7 \mathrm{~b}$ & $2.5 \mathrm{~b}$ & $25.8 \mathrm{a}$ & $15.6 \mathrm{a}$ \\
\hline $40\left(\mathrm{~N}_{1}\right)$ & & $240.0 \mathrm{a}$ & $10.9 \mathrm{a}$ & $4.5 \mathrm{a}$ & $14.2 \mathrm{~b}$ & $9.6 \mathrm{~b}$ \\
\hline $60\left(\mathrm{~N}_{2}\right)$ & & $246.1 \mathrm{a}$ & $11.3 \mathrm{a}$ & $4.3 \mathrm{a}$ & $14.6 \mathrm{~b}$ & $9.6 \mathrm{~b}$ \\
\hline $80\left(\mathrm{~N}_{3}\right)$ & & $247.1 \mathrm{a}$ & $11.1 \mathrm{a}$ & $4.5 \mathrm{a}$ & $12.5 \mathrm{~b}$ & $8.7 \mathrm{~b}$ \\
\hline & $0\left(\mathrm{~F}_{0}\right)$ & $203.8 \mathrm{a}$ & $8.9 \mathrm{a}$ & $3.9 \mathrm{a}$ & $17.6 \mathrm{a}$ & $10.6 \mathrm{a}$ \\
\hline & $100\left(\mathrm{~F}_{1}\right)$ & $201.6 \mathrm{a}$ & $9.6 \mathrm{a}$ & $4.1 \mathrm{a}$ & $16.8 \mathrm{a}$ & $10.9 \mathrm{a}$ \\
\hline & $200\left(\mathrm{~F}_{2}\right)$ & $199.9 \mathrm{a}$ & $9.2 \mathrm{a}$ & $3.9 \mathrm{a}$ & $15.9 \mathrm{a}$ & $11.1 \mathrm{a}$ \\
\hline \multicolumn{7}{|c|}{ Winter $2018 / 2019$} \\
\hline $0\left(\mathrm{~N}_{0}\right)$ & & $212.0 \mathrm{c}$ & $11.3 \mathrm{~b}$ & $3.7 \mathrm{~b}$ & $12.7 \mathrm{a}$ & $11.0 \mathrm{a}$ \\
\hline $40\left(\mathrm{~N}_{1}\right)$ & & $326.0 \mathrm{a}$ & $14.1 \mathrm{a}$ & $4.1 \mathrm{a}$ & $14.0 \mathrm{a}$ & $10.7 \mathrm{a}$ \\
\hline $60\left(\mathrm{~N}_{2}\right)$ & & $263.0 \mathrm{~b}$ & $16.1 \mathrm{a}$ & $4.2 \mathrm{a}$ & $9.3 \mathrm{~b}$ & $11.3 \mathrm{a}$ \\
\hline \multirow[t]{4}{*}{$80\left(\mathrm{~N}_{3}\right)$} & & $284.4 \mathrm{ab}$ & $15.8 \mathrm{a}$ & $4.2 \mathrm{a}$ & $9.9 \mathrm{~b}$ & $10.8 \mathrm{a}$ \\
\hline & $0\left(\mathrm{~F}_{0}\right)$ & $287.1 \mathrm{a}$ & $15.0 \mathrm{a}$ & $4.1 \mathrm{a}$ & $11.4 \mathrm{a}$ & $10.6 \mathrm{a}$ \\
\hline & $100\left(\mathrm{~F}_{1}\right)$ & $260.6 \mathrm{~b}$ & $13.7 \mathrm{~b}$ & $4.1 \mathrm{a}$ & $11.3 \mathrm{a}$ & $11.3 \mathrm{a}$ \\
\hline & $200\left(F_{2}\right)$ & $266.4 \mathrm{ab}$ & $14.3 \mathrm{ab}$ & $3.9 \mathrm{~b}$ & $11.8 \mathrm{a} \mathrm{b}$ & $10.9 \mathrm{a}$ \\
\hline
\end{tabular}

*values marked with alphabetical letter (s), within a comparable group of means, do not significantly differ, using revised L.S.D test at 0.05 level. 
Table 7. Inner head stem characters of cabbage as affected by the interaction between nitrogen fertilizer rates and fulvic acid concentrations in the winter seasons of 2017/2018 and 2018/2019.

\begin{tabular}{|c|c|c|c|c|c|c|}
\hline \multicolumn{2}{|c|}{ Treatments } & \multicolumn{5}{|c|}{ Inner head stem characters } \\
\hline $\begin{array}{l}\text { Nitrogen fertilizer } \\
\left(\mathrm{kg} \mathrm{fed}^{-1}\right)\end{array}$ & $\begin{array}{c}\text { Fulvic acid } \\
(\mathbf{p p m})\end{array}$ & $\begin{array}{l}\text { Fresh Wt. } \\
\text { (g) }\end{array}$ & $\begin{array}{c}\text { Length } \\
\text { (cm) }\end{array}$ & $\begin{array}{l}\text { Diameter } \\
(\mathbf{c m})\end{array}$ & $\begin{array}{c}\text { \% Head } \\
\text { Wt. }\end{array}$ & $\begin{array}{c}\text { DM content } \\
(\%)\end{array}$ \\
\hline \multicolumn{7}{|c|}{ Winter $2017 / 2018$} \\
\hline \multirow{4}{*}{$0\left(\mathrm{~N}_{0}\right)$} & $0\left(\mathrm{~F}_{0}\right)$ & $72.7 \mathrm{c}$ & $3.1 \mathrm{~b}$ & $2.3 \mathrm{e}$ & $28.6 \mathrm{a}$ & $14.9 \mathrm{a}$ \\
\hline & $100\left(\mathrm{~F}_{1}\right)$ & $73.8 \mathrm{c}$ & $3.8 \mathrm{~b}$ & $2.6 \mathrm{de}$ & $25.2 \mathrm{ab}$ & $15.8 \mathrm{a}$ \\
\hline & $200\left(\mathrm{~F}_{2}\right)$ & $75.2 \mathrm{c}$ & $4.2 \mathrm{~b}$ & $2.7 \mathrm{~d}$ & $23.4 \mathrm{~b}$ & $16.0 \mathrm{a}$ \\
\hline & $0\left(\mathrm{~F}_{0}\right)$ & $230.4 \mathrm{ab}$ & $10.9 \mathrm{a}$ & $4.6 \mathrm{ab}$ & $13.5 \mathrm{c}$ & $9.3 \mathrm{~b}$ \\
\hline \multirow[t]{3}{*}{$40\left(\mathrm{~N}_{1}\right)$} & $100\left(\mathrm{~F}_{1}\right)$ & $229.8 \mathrm{ab}$ & $11.5 \mathrm{a}$ & $4.4 \mathrm{abc}$ & $15.2 \mathrm{c}$ & $9.8 \mathrm{~b}$ \\
\hline & $200\left(\mathrm{~F}_{2}\right)$ & $259.8 \mathrm{a}$ & $10.4 \mathrm{a}$ & $4.3 \mathrm{bc}$ & $13.8 \mathrm{c}$ & $9.6 \mathrm{~b}$ \\
\hline & $0(\mathrm{~F} 0)$ & $252.6 \mathrm{ab}$ & $11.5 \mathrm{a}$ & $4.3 \mathrm{bc}$ & $15.2 \mathrm{c}$ & $9.7 \mathrm{~b}$ \\
\hline \multirow[t]{3}{*}{$60\left(\mathrm{~N}_{2}\right)$} & $100\left(\mathrm{~F}_{1}\right)$ & $241.6 \mathrm{ab}$ & $11.4 \mathrm{a}$ & $4.4 \mathrm{abc}$ & $14.8 \mathrm{c}$ & $9.5 \mathrm{~b}$ \\
\hline & $200\left(\mathrm{~F}_{2}\right)$ & $244.3 \mathrm{ab}$ & $11.2 \mathrm{a}$ & $4.3 \mathrm{bc}$ & $13.8 \mathrm{c}$ & $9.7 \mathrm{~b}$ \\
\hline & $0\left(\mathrm{~F}_{0}\right)$ & $259.6 \mathrm{a}$ & $10.5 \mathrm{a}$ & $4.6 \mathrm{abc}$ & $12.9 \mathrm{c}$ & $8.4 \mathrm{~b}$ \\
\hline \multirow{2}{*}{$80\left(\mathrm{~N}_{3}\right)$} & $100\left(\mathrm{~F}_{1}\right)$ & $261.3 \mathrm{a}$ & $11.8 \mathrm{a}$ & $4.8 \mathrm{a}$ & $12.0 \mathrm{c}$ & $8.7 \mathrm{~b}$ \\
\hline & $200\left(\mathrm{~F}_{2}\right)$ & $220.4 \mathrm{~b}$ & $11.1 \mathrm{a}$ & $4.2 \mathrm{c}$ & $12.6 \mathrm{c}$ & $9.2 \mathrm{~b}$ \\
\hline \multicolumn{7}{|c|}{ Winter 2018 / 2019} \\
\hline \multirow{4}{*}{$0\left(\mathrm{~N}_{0}\right)$} & $0\left(\mathrm{~F}_{0}\right)$ & 227.4 ef & $11.1 \mathrm{~g}$ & $3.8 \mathrm{c}$ & $12.8 \mathrm{~b}$ & $10.9 \mathrm{ab}$ \\
\hline & $100\left(\mathrm{~F}_{1}\right)$ & $204.2 \mathrm{f}$ & $11.8 \mathrm{fg}$ & $3.9 \mathrm{bc}$ & $12.0 \mathrm{bc}$ & $11.2 \mathrm{ab}$ \\
\hline & $200\left(\mathrm{~F}_{2}\right)$ & $204.3 \mathrm{f}$ & $11.0 \mathrm{~g}$ & $3.4 \mathrm{~d}$ & $13.2 \mathrm{~b}$ & $10.9 \mathrm{ab}$ \\
\hline & $0\left(\mathrm{~F}_{0}\right)$ & $338.2 \mathrm{ab}$ & $15.3 \mathrm{bcd}$ & $4.2 \mathrm{ab}$ & $13.3 \mathrm{~b}$ & $9.8 \mathrm{~b}$ \\
\hline \multirow{2}{*}{$40\left(\mathrm{~N}_{1}\right)$} & $100\left(\mathrm{~F}_{1}\right)$ & $297.0 \mathrm{bc}$ & $13.3 \mathrm{ef}$ & $3.4 \mathrm{~d}$ & $13.4 \mathrm{ab}$ & $11.2 \mathrm{ab}$ \\
\hline & $200\left(\mathrm{~F}_{2}\right)$ & $342.9 \mathrm{a}$ & $13.6 \mathrm{de}$ & $3.9 \mathrm{bc}$ & $15.4 \mathrm{a}$ & $10.9 \mathrm{ab}$ \\
\hline \multirow{3}{*}{$60\left(\mathrm{~N}_{2}\right)$} & $0\left(\mathrm{~F}_{0}\right)$ & $276.2 \mathrm{~cd}$ & $17.3 \mathrm{a}$ & $4.1 \mathrm{abc}$ & $9.1 \mathrm{~d}$ & $10.5 \mathrm{~b}$ \\
\hline & $100\left(\mathrm{~F}_{1}\right)$ & $267.8 \mathrm{cde}$ & $15.1 \mathrm{bcd}$ & $4.2 \mathrm{ab}$ & $9.7 \mathrm{~d}$ & $12.1 \mathrm{a}$ \\
\hline & $200\left(\mathrm{~F}_{2}\right)$ & $245.1 \mathrm{def}$ & $15.8 \mathrm{abc}$ & $4.1 \mathrm{abc}$ & $9.2 \mathrm{~d}$ & $11.3 \mathrm{ab}$ \\
\hline \multirow{3}{*}{$80\left(\mathrm{~N}_{3}\right)$} & $0\left(\mathrm{~F}_{0}\right)$ & $306.6 \mathrm{abc}$ & $16.1 \mathrm{abc}$ & $4.3 \mathrm{a}$ & $10.4 \mathrm{~cd}$ & $10.9 \mathrm{ab}$ \\
\hline & $100\left(\mathrm{~F}_{1}\right)$ & $273.3 \mathrm{~cd}$ & 14.7 cde & $4.1 \mathrm{abc}$ & $10.0 \mathrm{~cd}$ & $10.6 \mathrm{ab}$ \\
\hline & $200\left(\mathrm{~F}_{2}\right)$ & $273.3 \mathrm{~cd}$ & $16.6 \mathrm{ab}$ & $4.1 \mathrm{abc}$ & $9.3 \mathrm{~d}$ & $10.6 \mathrm{ab}$ \\
\hline
\end{tabular}

*values marked with alphabetical letter (s), within a comparable group of means, do not significantly differ, using revised L.S.D test at 0.05 level.

Also, the result indicated that the three FA concentrations $\left(\mathrm{F}_{0}, \mathrm{~F}_{1}\right.$ and $\left.\mathrm{F}_{2}\right)$ were not differed in their effects on the studied characters in the first season. However, the results of the second season indicated that $F_{2}$, significantly, decreased stem diameter. The results illustrating the effects of the interaction between the levels of $\mathrm{N}$ fertilizer and fulvic acid in the two seasons, on the tested characters of the inner head stem, are shown in Table (7). The highest mean values of fresh weight, length and diameter of stem $(261.3 \mathrm{gm}, 11.8 \mathrm{~cm}$ and $4.8 \mathrm{~cm})$, respectively, were attained with $\mathrm{N}_{3} \mathrm{~F}_{1}(80$ $\mathrm{kg} \mathrm{N} / \mathrm{fed}+100 \mathrm{ppm} \mathrm{FA}$ ). Meanwhile, the percentages of the stem to head weight and its dry matter content, statistically, decreased with increasing of $\mathrm{N}$ application rate, irrespective of the applied level of fulvic acid, the lowest mean values were reported at $\mathrm{N}_{3} \mathrm{~F}_{1}$ treatment combination. Regarding the results of the second season, the comparisons among 12 treatment combinations clarifieded that fresh weight and length of inner head stem ,significantly, increased with the application of $\mathrm{N}_{1}, \mathrm{~N}_{2}$ and $\mathrm{N}_{3}$ together with any of the studied fulvic acid concentrations as compared with those of unfertilized with $\mathrm{N}\left(\mathrm{N}_{0} \mathrm{~F}_{0}, \mathrm{~N}_{0} \mathrm{~F}_{1}\right.$ and $\left.\mathrm{N}_{0} \mathrm{~F}_{2}\right)$.In addition, although stem diameter and dry matter contents were significantly affected by the present interaction, the effects of various treatment combinations were neither clear nor constant. Cabbage yield and quality characters

Concerning the effects of $\mathrm{N}$ fertilizer rates, fulvic acid concentrations and their interactions on total and marketable yield as well as fresh weight of the aboveground parts of cabbage plant, the results of the twogrowing season (Tables 8-9), generally, revealed that increasing the level of applied $\mathrm{N}$ fertilizer from N0 up to $\mathrm{N} 3 \quad(80 \mathrm{~kg} \mathrm{~N} / \mathrm{fed}$.) was associated with consistent increases in these characters. However, the three $\mathrm{N}$ levels $\left(\mathrm{N}_{1}, \mathrm{~N}_{2}\right.$ and $\left.\mathrm{N}_{3}\right)$ in the first season, and the highest $\mathrm{N}$ two levels $\left(\mathrm{N}_{2}\right.$ and $\left.\mathrm{N}_{3}\right)$, in the second season, were not 
significantly differed in their effects on total and marketable head yield as well as plant fresh weigh. On the contrary, nitrogen use efficiency (NUE) of cabbage plants, either estimated as $\mathrm{kg}$ head or $\mathrm{kg}$ plant/1 kg N, significantly decreased with increasing $\mathrm{N}$ fertilizer rate up to $\mathrm{N}_{3}(80 \mathrm{~kg} / \mathrm{fed})$, in the first season. The present results, also in harmony with those obtained by EL-Afifi et al. (2002), Haque et al. (2015), Riad et al. (2009), Hassan and El-Kader. (2016) and Yshiwas (2017), who found that increasing the rate of applied $\mathrm{N}$ rate caused statistical increases in total and marketable head yield as well as plant fresh weight until the rate of $80 \mathrm{~kg} / \mathrm{fed}$, $350 \mathrm{~kg} / \mathrm{ha}, 90 \mathrm{~kg} / \mathrm{fed}$ and $150 \mathrm{~kg} / \mathrm{ha}$, respectively. Meanwhile, reverse results were obtained, in the second season. The results of the first season indicated that the tested concentrations of fulvic acid $\left(\mathrm{F}_{0}, \mathrm{~F}_{1}\right.$ and $\left.\mathrm{F}_{2}\right)$ did not significantly differ in their effects on total and marketable yields and NUE in the first season. On the other hand, the marketable head yield and plant fresh weight, which were significantly decreased with application of FA at rates of $F_{1}$ and $F_{2}$ (100 and 200 $\mathrm{ppm}$ ) in the second season. The highest mean values on the total and marketable yields and plant fresh weight were recorded with treatment combinations of $\mathrm{N}_{3} \mathrm{~F}_{1}$ and $\mathrm{N}_{3} \mathrm{~F}_{0}$, in the first and second seasons, respectively. As for the effects of the present interaction on NUE, the obtained results of the first season showed that, cabbage plants fertilized with $40 \mathrm{~kg} \mathrm{~N} / \mathrm{fed}$ and treated with fulvic acid at rate $200 \mathrm{ppm}$ recorded the highest significant increase in NUE comparing with the other treatment combinations. Meanwhile, the results of the second season showed that the treatment combinations containing $\mathrm{N}_{1}, \mathrm{~N}_{2}$ or $\mathrm{N}_{3}$ together with any rate of fulvic acid were not statistically differed in their effects on NUE. Except for $\mathrm{N}_{1} \mathrm{~F}_{2}$ in the first season and $\left(\mathrm{N}_{1} \mathrm{~F}_{2}\right.$, $\mathrm{N}_{2} \mathrm{~F}_{2}$ ), in the second season. Regarding the indirect effects of HS in improving yield and quality, it was reported that HS application increased soil enzyme activity and promoted the growth of rhizosphere microorganisms (Sellamuthu and Govindaswamy, 2003). In addition, there is a vital role of HS in enhancing the stability of soil aggregates and in reducing the disaggregating effect of wetting and drying cycles on soil structure. The formation of these aggregates was explained in terms of the formation of clay humic complexes through bridging polyvalent cations adsorbed on clay surfaces (Piccolo and Mbagwu, 1994).

\section{Chemical constituents of cabbage leaves}

Concerning the effects of $\mathrm{N}$ fertilizer rates on chemical components of cabbage leaves, the results (Table, 10) indicated that increasing the level of applied $\mathrm{N}$ fertilizer from 0 up to $80 \mathrm{~kg} \mathrm{~N} /$ fed, did not show any significant effects on the contents of $\mathrm{N}$ and $\mathrm{P}$ and total carbohydrate in the two seasons and total chlorophyll content in the first one. On the contrary, the leaves content of total chlorophyll, in the second season, constantly and significantly increased with increments of the applied $\mathrm{N}$ level up to $80 \mathrm{~kg} \mathrm{~N} / \mathrm{fed}\left(\mathrm{N}_{3}\right)$.

Table 8. Total and marketable head yield and nitrogen use efficiency (NUE) of cabbage plants as affected by nitrogen fertilizer rates and fulvic acid concentrations in the winter seasons of 2017/2018 and 2018/2019

\begin{tabular}{|c|c|c|c|c|c|c|}
\hline \multicolumn{2}{|c|}{ Treatments } & \multirow[b]{2}{*}{$\begin{array}{l}\text { Total yield } \\
\left(\text { ton fed }^{-1}\right)\end{array}$} & \multirow{2}{*}{$\begin{array}{c}\text { Marketable } \\
\text { head yield } \\
\left(\text { ton fed }^{-1}\right)\end{array}$} & \multirow{2}{*}{$\begin{array}{c}\text { Plant fresh } \\
\text { wt. } \\
\text { (g) }\end{array}$} & \multirow{2}{*}{$\begin{array}{c}\text { NUE } \\
\left(k^{\prime} \text { head }\right. \\
\left.\text { kg }^{-1} \mathbf{N}\right)\end{array}$} & \multirow{2}{*}{$\begin{array}{c}\text { NUE } \\
\left(k^{2} \text { plant }\right. \\
\left.\text { kg-1 }^{-1}\right)\end{array}$} \\
\hline $\begin{array}{c}\text { Nitrogen fertilizer rates } \\
\left(\mathrm{kg} \mathrm{fed}^{-1}\right)\end{array}$ & $\begin{array}{c}\text { Fulvic acid } \\
(\mathrm{ppm})\end{array}$ & & & & & \\
\hline & & Winter & $2017 / 2018$ & & & \\
\hline $0\left(\mathrm{~N}_{0}\right)$ & & $3.515 \mathrm{c}$ & $1.700 \mathrm{~b}$ & $680 \mathrm{~b}$ & $0.229 \mathrm{c}$ & $0.501 \mathrm{c}$ \\
\hline $40\left(\mathrm{~N}_{1}\right)$ & & $16.139 \mathrm{~b}$ & $9.172 \mathrm{a}$ & $3037 \mathrm{a}$ & $35.141 \mathrm{a}$ & $58.889 \mathrm{a}$ \\
\hline $60\left(\mathrm{~N}_{2}\right)$ & & $18.123 \mathrm{ab}$ & $10.502 \mathrm{a}$ & $3135 \mathrm{a}$ & $24.894 \mathrm{~b}$ & $40.916 \mathrm{~b}$ \\
\hline \multirow[t]{4}{*}{$80\left(\mathrm{~N}_{3}\right)$} & & $21.248 \mathrm{a}$ & $12.255 \mathrm{a}$ & $3549 \mathrm{a}$ & $21.625 \mathrm{~b}$ & $35.862 \mathrm{~b}$ \\
\hline & $0\left(\mathrm{~F}_{0}\right)$ & $14.424 \mathrm{a}$ & $8.320 \mathrm{a}$ & $2540 \mathrm{a}$ & $20.822 \mathrm{a}$ & $34.965 \mathrm{a}$ \\
\hline & $100\left(\mathrm{~F}_{1}\right)$ & $14.860 \mathrm{a}$ & $8.336 \mathrm{a}$ & $2597 \mathrm{a}$ & $19.733 \mathrm{a}$ & $32.786 \mathrm{a}$ \\
\hline & $200\left(F_{2}\right)$ & $\begin{array}{r}14.990 \mathrm{a} \\
\text { Winter }\end{array}$ & $\begin{array}{r}8.566 \mathrm{a} \\
2018 / 2019\end{array}$ & $2663 \mathrm{a}$ & $20.862 \mathrm{a}$ & $34.373 \mathrm{a}$ \\
\hline $0\left(\mathrm{~N}_{0}\right)$ & & $23.723 \mathrm{c}$ & $11.982 \mathrm{c}$ & $3360 \mathrm{c}$ & $1.140 \quad \mathrm{~b}$ & $2.263 \mathrm{~b}$ \\
\hline $40\left(\mathrm{~N}_{1}\right)$ & & $27.718 \mathrm{~b}$ & $15.263 \mathrm{~b}$ & $4271 \mathrm{~b}$ & $16.170 \mathrm{a}$ & $22.772 \mathrm{a}$ \\
\hline $60\left(\mathrm{~N}_{2}\right)$ & & $30.325 \mathrm{ab}$ & $17.997 \mathrm{a}$ & $4800 \mathrm{a}$ & $19.275 \mathrm{a}$ & $24.011 \mathrm{a}$ \\
\hline \multirow[t]{4}{*}{$80\left(\mathrm{~N}_{3}\right)$} & & $31.734 \mathrm{a}$ & $18.435 \mathrm{a}$ & $4979 a$ & $15.979 \mathrm{a}$ & $20.250 \mathrm{a}$ \\
\hline & $0\left(\mathrm{~F}_{0}\right)$ & $29.705 \mathrm{a}$ & $16.989 \mathrm{a}$ & $4526 \mathrm{a}$ & $14.384 \mathrm{a}$ & $18.061 \mathrm{ab}$ \\
\hline & $100\left(\mathrm{~F}_{1}\right)$ & $27.369 \mathrm{~b}$ & $15.328 \mathrm{~b}$ & $4235 \mathrm{~b}$ & $10.815 \mathrm{~b}$ & $14.359 \mathrm{~b}$ \\
\hline & $200\left(F_{2}\right)$ & $28.052 \mathrm{ab}$ & $15.441 \mathrm{~b}$ & $4296 \mathrm{~b}$ & $14.224 \mathrm{a}$ & $19.551 \mathrm{a}$ \\
\hline
\end{tabular}

*values marked with alphabetical letter (s), within a comparable group of means, do not significantly differ, using revised L.S.D test at 0.05 level. 
Table 9. Total and marketable head yield and nitrogen use efficiency (NUE) of cabbage plants as affected by the interaction between nitrogen fertilizer rate and fulvic acid concentrations in the winter seasons of 2017/2018 and 2018/2019.

\begin{tabular}{|c|c|c|c|c|c|c|}
\hline \multicolumn{2}{|c|}{ Treatments } & \multirow[b]{2}{*}{$\begin{array}{l}\text { Total yield } \\
\left.\text { (ton fed }^{-1}\right)\end{array}$} & \multirow[b]{2}{*}{$\begin{array}{c}\text { Marketable } \\
\text { head yield } \\
\left(\text { ton } \text { fed }^{-1}\right)\end{array}$} & \multirow[b]{2}{*}{$\begin{array}{c}\text { Plant } \\
\text { fresh wt. } \\
\text { (g) }\end{array}$} & \multirow[b]{2}{*}{$\begin{array}{c}\text { NUE } \\
\left(k^{-1} \text { head kg-1 N) }\right.\end{array}$} & \multirow[b]{2}{*}{$\begin{array}{c}\text { NUE } \\
\left(\mathrm{kg}^{2} \text { plant kg } \mathrm{kg}^{-1} \mathrm{~N}\right)\end{array}$} \\
\hline $\begin{array}{l}\text { Nitrogen } \\
\text { fertilizer } \\
\left(\mathbf{k g ~ f e d}^{-1}\right)\end{array}$ & $\begin{array}{l}\text { Fulvic acid } \\
\quad(\mathbf{p p m})\end{array}$ & & & & & \\
\hline \multicolumn{7}{|c|}{ Winter $2017 / 2018$} \\
\hline \multirow{4}{*}{$0\left(\mathrm{~N}_{0}\right)$} & $0\left(\mathrm{~F}_{0}\right)$ & $3.1 \mathrm{f}$ & $1.488 \mathrm{e}$ & $575 \mathrm{e}$ & $0.196 \mathrm{e}$ & $0.424 \mathrm{~d}$ \\
\hline & $100\left(\mathrm{~F}_{1}\right)$ & $3.5 \mathrm{f}$ & $1.552 \mathrm{e}$ & $706 \mathrm{e}$ & $0.220 \mathrm{e}$ & $0.520 \mathrm{~d}$ \\
\hline & $200\left(\mathrm{~F}_{2}\right)$ & $3.9 \mathrm{f}$ & $2.058 \mathrm{e}$ & $758 \mathrm{e}$ & $0.272 \mathrm{e}$ & $0.558 \mathrm{~d}$ \\
\hline & $0\left(\mathrm{~F}_{0}\right)$ & $16.1 \mathrm{e}$ & $8.93 \mathrm{~d}$ & $3060 \mathrm{~cd}$ & $35.356 \mathrm{ab}$ & $61.995 \mathrm{a}$ \\
\hline \multirow[t]{3}{*}{$40\left(\mathrm{~N}_{1}\right)$} & $100\left(F_{1}\right)$ & $16.1 \mathrm{e}$ & $9.025 \mathrm{~d}$ & $2800 \mathrm{~d}$ & $31.378 \mathrm{~b}$ & $52.356 \mathrm{ab}$ \\
\hline & $200\left(\mathrm{~F}_{2}\right)$ & $16.2 \mathrm{e}$ & $9.563 \mathrm{~cd}$ & $3251 \mathrm{bc}$ & $38.687 \mathrm{a}$ & $62.314 \mathrm{a}$ \\
\hline & $0\left(\mathrm{~F}_{0}\right)$ & $17.1 \mathrm{de}$ & $10.323 \mathrm{bcd}$ & $3035 \mathrm{~cd}$ & $25.585 \mathrm{c}$ & $40.996 \mathrm{c}$ \\
\hline \multirow[t]{3}{*}{$60\left(\mathrm{~N}_{2}\right)$} & $100\left(\mathrm{~F}_{1}\right)$ & 17.7 cde & $10.038 \mathrm{bcd}$ & $3092 \mathrm{~cd}$ & $24.068 \mathrm{~cd}$ & $39.757 \mathrm{c}$ \\
\hline & $200\left(\mathrm{~F}_{2}\right)$ & $19.6 \mathrm{bcd}$ & $11.147 \mathrm{abc}$ & $3278 \mathrm{bc}$ & $25.027 \mathrm{~cd}$ & $41.996 \mathrm{bc}$ \\
\hline & $0\left(\mathrm{~F}_{0}\right)$ & $21.4 \mathrm{ab}$ & $12.54 \mathrm{a}$ & $3491 \mathrm{ab}$ & $22.15 \mathrm{~cd}$ & $36.447 \mathrm{c}$ \\
\hline \multirow[t]{4}{*}{$80\left(\mathrm{~N}_{3}\right)$} & $100\left(\mathrm{~F}_{1}\right)$ & $22.2 \mathrm{a}$ & $12.73 \mathrm{a}$ & $3787 \mathrm{a}$ & $23.265 \mathrm{~cd}$ & $38.516 \mathrm{c}$ \\
\hline & $200\left(\mathrm{~F}_{2}\right)$ & $20.2 \mathrm{abc}$ & $11.495 \mathrm{ab}$ & $3367 \mathrm{bc}$ & $19.462 \mathrm{~d}$ & $32.624 \mathrm{c}$ \\
\hline & & & Winter 2018 & 2019 & & \\
\hline & $0\left(\mathrm{~F}_{0}\right)$ & $24.3 \mathrm{de}$ & $12.31 \mathrm{~cd}$ & $3488 \mathrm{f}$ & $1.197 \mathrm{~d}$ & $2.349 \mathrm{c}$ \\
\hline \multirow[t]{3}{*}{$0\left(\mathrm{~N}_{0}\right)$} & $100\left(\mathrm{~F}_{1}\right)$ & 25.3 cde & $13.14 \mathrm{~cd}$ & $3410 \mathrm{f}$ & $1.188 \mathrm{~d}$ & $2.297 \mathrm{c}$ \\
\hline & $200\left(\mathrm{~F}_{2}\right)$ & $21.6 \mathrm{e}$ & $10.48 \mathrm{~d}$ & $3181 \mathrm{f}$ & $1.037 \mathrm{~d}$ & $2.142 \mathrm{c}$ \\
\hline & $0\left(\mathrm{~F}_{0}\right)$ & $29.9 \mathrm{~b}$ & $17.29 \mathrm{ab}$ & 4447 cde & $19.901 \mathrm{ab}$ & $23.98 \mathrm{ab}$ \\
\hline \multirow[t]{3}{*}{$40\left(\mathrm{~N}_{1}\right)$} & $100\left(\mathrm{~F}_{1}\right)$ & $25.5 \mathrm{cde}$ & $13.87 \mathrm{c}$ & $4099 \mathrm{e}$ & $11.112 \mathrm{c}$ & $17.216 \mathrm{~b}$ \\
\hline & $200\left(\mathrm{~F}_{2}\right)$ & $27.7 \mathrm{bcd}$ & $14.63 \mathrm{bc}$ & $4266 \mathrm{de}$ & $17.497 \mathrm{ab}$ & $27.121 \mathrm{a}$ \\
\hline & $0\left(\mathrm{~F}_{0}\right)$ & $29.9 \mathrm{~b}$ & $18.58 \mathrm{a}$ & $4933 \mathrm{ab}$ & $21.164 \mathrm{a}$ & $24.066 \mathrm{ab}$ \\
\hline \multirow[t]{3}{*}{$60\left(\mathrm{~N}_{2}\right)$} & $100\left(\mathrm{~F}_{1}\right)$ & $29.2 \mathrm{bc}$ & $17.16 \mathrm{ab}$ & $4677 \mathrm{bcd}$ & $16.355 \mathrm{abc}$ & $21.113 \mathrm{ab}$ \\
\hline & $200\left(\mathrm{~F}_{2}\right)$ & $31.7 \mathrm{ab}$ & $18.24 \mathrm{a}$ & 4792 bc & $20.307 \mathrm{ab}$ & $26.853 \mathrm{a}$ \\
\hline & $0\left(\mathrm{~F}_{0}\right)$ & $34.6 \mathrm{a}$ & $19.76 \mathrm{a}$ & $5236 \mathrm{a}$ & $15.275 \mathrm{abc}$ & $21.852 \mathrm{ab}$ \\
\hline \multirow{2}{*}{$80\left(\mathrm{~N}_{3}\right)$} & $100\left(\mathrm{~F}_{1}\right)$ & $29.5 \mathrm{bc}$ & $17.13 \mathrm{ab}$ & $4755 \mathrm{bc}$ & $14.606 \mathrm{bc}$ & $16.808 \mathrm{~b}$ \\
\hline & $200\left(\mathrm{~F}_{2}\right)$ & $31.1 \mathrm{ab}$ & $18.41 \mathrm{a}$ & $4947 \mathrm{ab}$ & $18.055 \mathrm{ab}$ & $22.087 \mathrm{ab}$ \\
\hline
\end{tabular}

*values marked with alphabetical letter (s), within a comparable group of means, do not significantly differ, using revised L.S.D test at 0.05 level.

The favorable effect of $\mathrm{N}$ on the leaves' chlorophyll content may be caused by delaying senility and break down chlorophyll in cabbage leaves (Barakat, 1987). The obtained results appeared to be in close agreement with those reported by Singh et al. (2001) and Freyman et al. (1991), who indicated that adequate $\mathrm{N}$ is necessary in the formation of chlorophyll and a component of proteins. The present result, also, in accordance with those obtained by Babik et al. (1996) on brussels sprouts, and El-Afifi et al. (2002) on cabbage, who pointed out that increasing $\mathrm{N}$ rate up to $600 \mathrm{~kg} / \mathrm{ha}$ and to $80 \mathrm{~kg} / \mathrm{fed}$, respectively, was positively correlated with the leaves chlorophyll content. Likewise, Tanaka and Sato (1997) illustrated that the concentration of phosphorus, potassium and magnesium in plant varied with $\mathrm{N}$ rate. The results of $\mathrm{FA}$ revealed that the leaves contents of $\mathrm{N}$ and $\mathrm{P}$ significantly decreased with the application of $F_{1}$ and $F_{2}$ compared with control treatment $\left(\mathrm{F}_{0}\right)$. Meanwhile, the content of total carbohydrate increased in the first season. The obtained results of the second season (Table, 10) revealed that the three levels of FA did not significantly differ in their effects on the leaves content of $\mathrm{N}, \mathrm{P}$ and total chlorophyll, while the highest significant increase of total carbohydrate was recorded with $\mathrm{F}_{1}$ (100 ppm FA). The total chlorophyll content was not responded to FA application. The results also, revealed that increasing the level of applied $\mathrm{N}$ rate up to $80 \mathrm{~kg} / \mathrm{fed}$ regardless the used rate of fulvic acid, progressively increased the content of total chlorophyll. The highest significant increase was attained with the treatment combination of $\mathrm{N}_{3} \mathrm{~F}_{0}$ (Table, 11). 


\section{CONCLUSION}

It is concluded that fertilizing cabbage plants with $\mathrm{N}$ at a rate of $80 \mathrm{~kg} \mathrm{~N} / \mathrm{fed}$ with $100 \mathrm{ppm} F A$ have the potential to be used for increasing high marketable yield with good quality of cabbage, under the prevailing condition of the present study and other similar regions.

Table 10. Chemical constituents of cabbage leaves as affected by nitrogen fertilizer rates and fulvic acid concentrations in the winter seasons of $2017 / 2018$ and $2018 / 2019$.

\begin{tabular}{|c|c|c|c|c|c|}
\hline \multicolumn{2}{|l|}{ Treatments } & \multirow{3}{*}{$\begin{array}{c}\mathrm{N} \\
(\%)\end{array}$} & \multirow{3}{*}{$\begin{array}{c}P \\
(\%)\end{array}$} & \multirow{3}{*}{$\begin{array}{c}\text { Total } \\
\text { carbohyd-rate } \\
(\%)\end{array}$} & \multirow{3}{*}{$\begin{array}{c}\text { Total } \\
\text { chlorophyll } \\
\text { (mg/100g fresh wt. }\end{array}$} \\
\hline Nitrogen fertilizer & & & & & \\
\hline $\begin{array}{c}\text { Rate } \\
\left(\text { kg fed }^{-1}\right)\end{array}$ & $\begin{array}{l}\text { Fulvic acid } \\
\text { (ppm) }\end{array}$ & & & & \\
\hline \multicolumn{6}{|c|}{ Winter $2017 / 2018$} \\
\hline $0\left(\mathrm{~N}_{0}\right)$ & & $1.72 \mathrm{a}$ & $0.53 \mathrm{a}$ & $10.43 \mathrm{~b}$ & $44.24 \mathrm{a}$ \\
\hline $40\left(\mathrm{~N}_{1}\right)$ & & $1.38 \mathrm{a}$ & $0.57 \mathrm{a}$ & $11.16 \mathrm{ab}$ & $35.64 \mathrm{a}$ \\
\hline $60\left(\mathrm{~N}_{2}\right)$ & & $1.70 \mathrm{a}$ & $0.53 \mathrm{a}$ & $11.77 \mathrm{a}$ & $37.42 \mathrm{a}$ \\
\hline $80\left(\mathrm{~N}_{3}\right)$ & & $1.38 \mathrm{a}$ & $0.58 \mathrm{a}$ & $10.90 \mathrm{ab}$ & $37.10 \mathrm{a}$ \\
\hline & $0\left(\mathrm{~F}_{0}\right)$ & $1.65 \mathrm{a}$ & $0.58 \mathrm{a}$ & $10.68 \mathrm{~b}$ & $39.71 \mathrm{a}$ \\
\hline & $100\left(\mathrm{~F}_{1}\right)$ & $1.50 \mathrm{~b}$ & $0.55 \mathrm{~b}$ & $11.31 \mathrm{a}$ & $37.53 \mathrm{a}$ \\
\hline & $200\left(\mathrm{~F}_{2}\right)$ & $1.60 \mathrm{~b}$ & $0.54 \mathrm{~b}$ & $11.18 \mathrm{ab}$ & $38.56 \mathrm{a}$ \\
\hline \multicolumn{6}{|c|}{ Winter 2018/2019 } \\
\hline $0\left(\mathrm{~N}_{0}\right)$ & & $1.82 \mathrm{a}$ & $0.65 \mathrm{a}$ & $10.60 \mathrm{a}$ & $20.18 \mathrm{c}$ \\
\hline $40\left(\mathrm{~N}_{1}\right)$ & & $1.79 \mathrm{a}$ & $0.73 \mathrm{a}$ & $11.10 \mathrm{a}$ & $25.69 \mathrm{~b}$ \\
\hline $60\left(\mathrm{~N}_{2}\right)$ & & $1.77 \mathrm{a}$ & $0.70 \mathrm{a}$ & $11.10 \mathrm{a}$ & $27.89 \mathrm{~b}$ \\
\hline $80\left(\mathrm{~N}_{3}\right)$ & & $1.71 \mathrm{a}$ & $0.67 \mathrm{a}$ & $10.51 \mathrm{a}$ & $33.62 \mathrm{a}$ \\
\hline & $0\left(\mathrm{~F}_{0}\right)$ & $1.75 \mathrm{a}$ & $0.69 \mathrm{a}$ & $10.72 \mathrm{~b}$ & $28.05 \mathrm{a}$ \\
\hline & $100\left(\mathrm{~F}_{1}\right)$ & $1.78 \mathrm{a}$ & $0.68 \mathrm{a}$ & $11.29 \mathrm{a}$ & $26.10 \mathrm{a}$ \\
\hline & $200\left(\mathrm{~F}_{2}\right)$ & $1.79 \mathrm{a}$ & $0.69 \mathrm{a}$ & $10.46 \mathrm{~b}$ & $26.32 \mathrm{a}$ \\
\hline
\end{tabular}

*values marked with alphabetical letter (s), within a comparable group of means, do not significantly differ, using revised L.S.D test at 0.05 level.

Table 11. Chemical constituents of cabbage leaves as affected by the interaction between nitrogen fertilizer rates and fulvic acid concentrations in the winter seasons of 2017/2018 and 2018/2019.

\begin{tabular}{|c|c|c|c|c|c|}
\hline \multicolumn{2}{|c|}{ Treatments } & \multirow[b]{2}{*}{$\begin{array}{l}\mathrm{N} \\
(\%)\end{array}$} & \multirow[b]{2}{*}{$\begin{array}{l}\mathbf{P} \\
(\%)\end{array}$} & \multirow{2}{*}{$\begin{array}{c}\text { Total } \\
\text { carbohydr-ate } \\
(\%) \\
\end{array}$} & \multirow{2}{*}{$\begin{array}{c}\text { Total } \\
\text { chlorophyll } \\
\text { (mg/100g fresh wt.) }\end{array}$} \\
\hline $\begin{array}{l}\text { Nitrogen fertilizer } \\
\quad\left(\mathrm{kg} \mathrm{fed}^{-1}\right)\end{array}$ & $\begin{array}{l}\text { Fulvic acid } \\
\text { (ppm) }\end{array}$ & & & & \\
\hline \multicolumn{6}{|c|}{ Winter $2017 / 2018$} \\
\hline \multirow{4}{*}{$0\left(\mathrm{~N}_{0}\right)$} & $0\left(\mathrm{~F}_{0}\right)$ & $1.787 \mathrm{ab}$ & $0.536 \mathrm{def}$ & $10.255 \mathrm{c}$ & $44.45 \mathrm{a}$ \\
\hline & $100\left(\mathrm{~F}_{1}\right)$ & $1.754 \mathrm{ab}$ & $0.548 \mathrm{~b}-\mathrm{e}$ & $10.863 \mathrm{bc}$ & $44.94 \mathrm{a}$ \\
\hline & $200\left(\mathrm{~F}_{2}\right)$ & $1.622 \mathrm{bcd}$ & $0.517 \mathrm{def}$ & $10.184 \mathrm{c}$ & $43.31 \mathrm{ab}$ \\
\hline & $0\left(\mathrm{~F}_{0}\right)$ & $1.465 \mathrm{cde}$ & $0.602 \mathrm{abc}$ & $10.916 \mathrm{bc}$ & $37.78 \mathrm{c}$ \\
\hline \multirow[t]{3}{*}{$40\left(\mathrm{~N}_{1}\right)$} & $100\left(\mathrm{~F}_{1}\right)$ & $1.291 \mathrm{e}$ & $0.579 \mathrm{a}-\mathrm{d}$ & $11.600 \mathrm{ab}$ & $34.11 \mathrm{c}$ \\
\hline & $200\left(F_{2}\right)$ & $1.397 \mathrm{e}$ & $0.539 \mathrm{def}$ & $10.979 \mathrm{bc}$ & $35.04 \mathrm{c}$ \\
\hline & $0\left(\mathrm{~F}_{0}\right)$ & $1.930 \mathrm{a}$ & $0.556 \mathrm{a}-\mathrm{e}$ & $10.624 \mathrm{bc}$ & $37.58 \mathrm{c}$ \\
\hline \multirow[t]{3}{*}{$60\left(\mathrm{~N}_{2}\right)$} & $100\left(\mathrm{~F}_{1}\right)$ & 1.504 cde & $0.543 \mathrm{c}-\mathrm{f}$ & $12.248 \mathrm{a}$ & $37.03 \mathrm{c}$ \\
\hline & $200\left(\mathrm{~F}_{2}\right)$ & $1.640 \mathrm{bc}$ & $0.484 \mathrm{f}$ & $12.301 \mathrm{a}$ & $37.65 \mathrm{c}$ \\
\hline & $0\left(\mathrm{~F}_{0}\right)$ & $1.421 \mathrm{cde}$ & $0.613 \mathrm{a}$ & $10.939 \mathrm{bc}$ & $39.01 \mathrm{bc}$ \\
\hline \multirow{2}{*}{$80\left(\mathrm{~N}_{3}\right)$} & $100\left(F_{1}\right)$ & $1.409 \mathrm{de}$ & 0.512 ef & $10.521 b c$ & $34.06 \mathrm{c}$ \\
\hline & $200\left(F_{2}\right)$ & $1.333 \mathrm{e}$ & $0.610 \mathrm{ab}$ & $11.241 \mathrm{abc}$ & $38.21 b c$ \\
\hline
\end{tabular}


Cont. Table 11. Chemical constituents of cabbage leaves as affected by the interaction between nitrogen fertilizer rates and fulvic acid concentrations in the winter seasons of 2017/2018 and 2018/2019.

\begin{tabular}{|c|c|c|c|c|c|}
\hline \multicolumn{2}{|c|}{ Treatments } & \multirow{2}{*}{$\begin{array}{l}\mathbf{N} \\
(\%)\end{array}$} & \multirow{2}{*}{$\begin{array}{c}\mathbf{P} \\
(\%)\end{array}$} & \multirow{2}{*}{$\begin{array}{c}\text { Total } \\
\text { carbohydr-ate } \\
(\%)\end{array}$} & \multirow{2}{*}{$\begin{array}{c}\text { Total } \\
\text { chlorophyll } \\
\text { (mg/100g fresh wt. }\end{array}$} \\
\hline $\begin{array}{c}\text { Nitrogen fertilizer } \\
\left(\mathrm{kg} \mathrm{fed}^{-1}\right)\end{array}$ & $\begin{array}{c}\text { Fulvic acid } \\
(\mathbf{p p m})\end{array}$ & & & & \\
\hline \multicolumn{6}{|c|}{ Winter 2018 / 2019} \\
\hline \multirow{4}{*}{$0\left(\mathrm{~N}_{0}\right)$} & $0\left(\mathrm{~F}_{0}\right)$ & $1.760 \mathrm{ab}$ & $0.697 \mathrm{abc}$ & $10.943 \mathrm{c}-\mathrm{f}$ & $19.0 \mathrm{~g}$ \\
\hline & $100\left(\mathrm{~F}_{1}\right)$ & $1.858 \mathrm{a}$ & $0.627 \mathrm{c}$ & $10.695 \mathrm{def}$ & $20.7 \mathrm{efg}$ \\
\hline & $200\left(\mathrm{~F}_{2}\right)$ & $1.851 \mathrm{a}$ & $0.629 \mathrm{c}$ & $10.162 \mathrm{efg}$ & $20.5 \mathrm{fg}$ \\
\hline & $0\left(\mathrm{~F}_{0}\right)$ & $1.848 \mathrm{a}$ & $0.685 a b c$ & $11.773 \mathrm{abc}$ & $27.3 \mathrm{bcd}$ \\
\hline $40\left(\mathrm{~N}_{1}\right)$ & $100\left(\mathrm{~F}_{1}\right)$ & $1.726 \mathrm{ab}$ & $0.714 a b c$ & $12.097 \mathrm{a}$ & $23.8 \mathrm{~d}-\mathrm{g}$ \\
\hline & $200\left(\mathrm{~F}_{2}\right)$ & $1.797 \mathrm{ab}$ & $0.778 \mathrm{a}$ & $9.385 \mathrm{~g}$ & $25.9 c-f$ \\
\hline & $0\left(\mathrm{~F}_{0}\right)$ & $1.718 \mathrm{ab}$ & $0.742 \mathrm{ab}$ & $9.989 \mathrm{fg}$ & $27.8 \mathrm{bcd}$ \\
\hline \multirow[t]{3}{*}{$60\left(\mathrm{~N}_{2}\right)$} & $100\left(\mathrm{~F}_{1}\right)$ & $1.830 \mathrm{ab}$ & $0.698 \mathrm{abc}$ & $11.960 \mathrm{ab}$ & $29.7 \mathrm{bc}$ \\
\hline & $200\left(F_{2}\right)$ & $1.773 \mathrm{ab}$ & $0.664 \mathrm{bc}$ & $11.298 \mathrm{a}-\mathrm{d}$ & 26.2 cde \\
\hline & $0\left(\mathrm{~F}_{0}\right)$ & $1.683 \mathrm{~b}$ & $0.639 \mathrm{c}$ & $10.157 \mathrm{efg}$ & $38.1 \mathrm{a}$ \\
\hline \multirow{2}{*}{$80\left(\mathrm{~N}_{3}\right)$} & $100\left(\mathrm{~F}_{1}\right)$ & $1.720 \mathrm{ab}$ & $0.690 \mathrm{abc}$ & $10.388 \mathrm{def}$ & $30.2 \mathrm{bc}$ \\
\hline & $200\left(\mathrm{~F}_{2}\right)$ & $1.742 \mathrm{ab}$ & $0.676 \mathrm{bc}$ & $10.988 \mathrm{~b}-\mathrm{e}$ & $32.6 \mathrm{ab}$ \\
\hline
\end{tabular}

*values marked with alphabetical letter (s), within a comparable group of means, do not significantly differ, using revised L.S.D test at 0.05 level.

\section{REFERENCES}

A.O.A.C. 1992. Official and tentative methods of analysis. $12^{\text {th }}$ Ed. Washington, D. C., U.S.A.

Babik, I., J. Rumpel and K. Elkner.1996. The influence of nitrogen fertilization on yield, quality and senescence of Brussels sprouts. Acta Horticulturae.407, 353-360.

Barakat, M. A. S. 1987. The effect of nitrogen, potassium and chlormequate on the yield and the quality of seed potato. Ph. D. Thesis, Fac. of Agric., Alexandria Univ., Egypt.

Boyhan, G. E., W. M. Randle, A. C. Purvis, P. M. Lewis, D. O. Linton, R. L.Torrance and D.E. Curry. 2001. Evaluation of growth stimulants on short-day onions. HortTechnology. 11(1), 38-42.

Chen, Y., M.De. Nobili and T. Aviad. 2004. Stimulatory effects of humic substances on plant growth. Soil organic matter in sustainable agriculture, CRC Press, Washington. 103-130.

Din, M., M.Qasim and M. Alam.2007. Effect of different levels of $\mathrm{N}, \mathrm{P}$ and $\mathrm{K}$ on the growth and yield of cabbage. Journal of Agricultural Research (Pakistan).45(2), 171176.

Duval, J. R., F. J. Dainello, V. A. Haby and D. R. Earhart. 1998. Evaluating leonardite as a crop growth enhancer for turnip and mustard greens. HortTechnology. 8(4), 564567.

Evenhuis, B.1976. Simplified methods for foliar analysis parts.I.VII. International Report. Royal Tropical. Institute, Amestrdam.

El-Afifi, S.T., M.M. Zaghloul and E. I. Ibrahim. 2002. Effect of transplant age, nitrogen fertilizer levels and biofertilization on cabbage. 2nd Inter. Conf. Hort. Sci., 1012 Sept. Kafr, Tanta Univ., Egypt.
FAO.STAT.2018. Online statistical database. In: FAOSTAT [online]. http://faostat.fao.org

Feibert, E.B., C.C. Shock and L. D. Saunders.2003. Nonconventional additives leave onion yield and quality unchanged. HortScience. 38(3), 381-386.

Freyman, S., P. M. Toivonen, W.C. Lin, P. W. Perrin and J. W. Hall. 1991. Effect of nitrogen fertilization on yield, storage losses and chemical composition of winter cabbage. Canadian journal of plant science. 71(3), 943946.

Haque, F.A., N. Islam, M.N. Islam, A. Ullah and M.D. Sarkar. 2015. Growth, yield and profitability of cabbage (Brassica oleracea L.) as influenced by applied nitrogen and plant spacing. The Agriculturists. 13(1), 35-45.

Hartz, T.K and T. G. Bottoms. 2010. Humic substances generally ineffective in improving vegetable crop nutrient uptake or productivity. HortScience.45(6), 906-910.

Hassan, S.M and D.Y. Abd El-Kader. 2016.Influence of Starter Fertilizer and Calcium Nitrate Rates on Vegetative Growth, Yield and Nutritional Quality of Cabbage. Alexandria Science Exchange Journal.37(4):811-819.

Hewett, E.W. 2006. An overview of preharvest factors influencing postharvest quality of horticultural products. International Journal of Postharvest Technology and Innovation.1(1):4-15.

Hsieh, C. F., H. C. Fang, K.Nan and K. N. Hsu. 1995. Effect of continuous use of organic manures on the growth and yield of vegetable soybean' and cabbage. Bulletin of Taichung District. Agric. Improvement Sta., Japan. 46, 110. 
Manchali, S., N. Kotamballi, M.Chidambara and S.P. Bhimanagouda. 2012. Crucial facts about health benefits of popular cruciferous vegetables. Journal of Functional Foods. 4(1), 94-106. https://doi.org/10.1016/j.jff.2011.08.004.

Manea, A. I. 2017. Fertilizer type on cabbage growth and yield. International Journal of Vegetable Science. 23(6), 567-574.

Marschner, H .1995. Mineral nutrition of higher plants (2nd ed., p. 864). Acad Press Limited, Text Book.

Murphy, J and J.P.Riley. 1962. A modified single solution method for the determination of phosphorus in natural water. Anal. Chim. Acta. 27:31-36.

Patrick, R., A.Lesaca and B.T. Anabella.2012. Increasing cabbage yield through SSNM. Bar Digest research and development. Planning and Economic Development of East Gojjam Zone (2004).

Piccolo, A and J. S. C. Mbagwu. 1994. Humic substances and surfactants effects on the stability of two tropical soils. Soil Science Society of America Journal, 58(3), 950-955.

Riad, G., A. Ghoname, A. Ahmed, M. El-Baky and A. Hegazi .2009. Cabbage nutritional quality as influenced by planting density and nitrogen fertilization. Fruit, Vegetable and Cereal Science and Biotechnology. 3(1):68-74.

Russell, E. W. 1973. Soil conditions and plant growth (10th Ed.). London: Longman, pp. 849.

Schmidt, W., S. Santi, R.Pinton and Z.Varanini. 2007. Waterextractable humic substances alter root development and epidermal cell pattern in Arabidopsis. Plant and Soil, 300(1-2), 259-267.

Selim, E. M and A. A. Mosa. 2012. Fertigation of humic substances improves yield and quality of broccoli and nutrient retention in a sandy soil. Journal of Plant Nutrition and Soil Science. 175(2), 273-281.

Sellamuthu, K.M and M. Govindaswamy. 2003. Effect of fertiliser and humic acid on rhizosphere microorganisms and soil enzymes at an early stage of sugarcane growth. Sugar Tech. 5(4), 273-277.
Singh, M. 2008. Influence of triacontanol, nitrogen and phosphorus on the growth, yield and quality of ginger (Zingiber officinale Rosc) and turmeric (Curcuma longa L.). Doctoral dissertation, Aligarh Muslim University, Aligarh, India.

Singh, R. V., A. N. Maurya and K. P. Singh. 2001. Response of F1 hybrid cabbage to nitrogen and phosphorus fertilization. Veg. Sci. 28(1), 48-50.

Snedecor, G. W and W. G. Cochron.1980. Statistical Method $7^{\text {th }}$ ed. Iowa State University Press, Ames,pp.507.

Tanaka, T and T. Sato. 1997. Growth and nutrient absorption characteristics of three commercial cabbage cultivars under varying nitrogen levels. Japanese Journal of Soil Science and Plant Nutrition. 68(5), 493-500.

Tindall, H. D. 1983. Vegetables in the Tropics. MacMillan International Higher Education Ltd, London, 534 pages, Houndmills. London [Cited from Hort. Abstr., 50(6): 3791-1984].

Tindall, M. 2000. Mineral and organic fertilizing in cabbage. Residual effect for commercial cultivation on yield and quality performance with organic farming. Hort. Bras, 6(1), 15-20.

Van Hees, P. A., D. L.Jones, R. Finlay, D. L. Godbold and U. S. Lundström. 2005. The carbon we do not see-the impact of low molecular weight compounds on carbon dynamics and respiration in forest soils: a review. Soil Biology and Biochemistry, 37(1), 1-13.

Verma, H and D.D. Nawange. 2015. Effect of different levels of nitrogen and Sulphur on the growth, yield and quality of cabbage [Brassica Oleracea Var. Capitata L.]. Agric. Sci. Digest. 35 (2): 152-154.

Witham, F. H., D. F. Blaydes and R. M. Devlin. 1971. Experiments in plant physiology, Van Nostrand Reinhold Co., New York (N.Y.).

Yagodin, B. A. 1984. Agricultural Chemistry (1st ed). Mir Publishers, Moscow.

Yshiwas, Y. 2017. Effect of different rate of nitrogen fertilizer on the growth and yield of cabbage (Brassica oleraceae) at Debre Markos, North West Ethiopia. Afr. J. Plant Sci., 11(7), 276-281. 


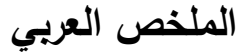

\section{تأثير الأسمدة النيتروجينية وحمض الفولفيك على النمو والإنتاجية والجودة الغذائية للكرنب}

أشرف محمود، على حسن عبد الرازق، سناء مرسى العربي، شيماء محمد رجب

الأوراق الخارجية ووزنها الرطب بينما خفضت النسبة المئويـة

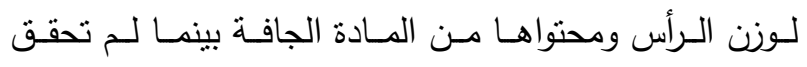

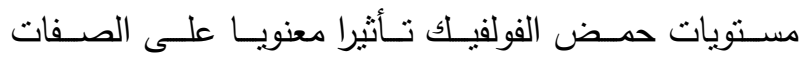

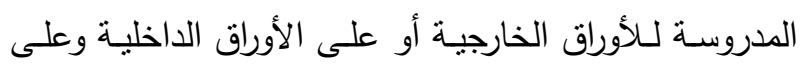
صفات الرأس. وأظهرت التأثير المتداخل ببين العاملين بأن

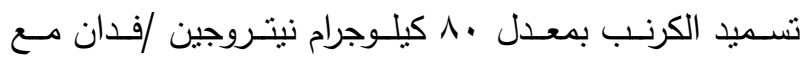
المعدل . . 1 جزء فى المليون من حمض الفولفيك هى أفضل

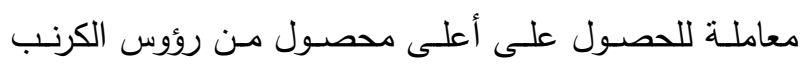
القابلة للتسويق والمناسبة للسوق المحلى وذلك تحت الظروف المماثلة لظروف الدراسة الحالية والمناطق المشابهة. الكلمـات المفتاحية: كرنب، التسـميد النيتروجينى، فولفيك

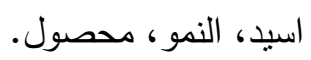

إجريـت تجربتـان حقليتـان خـلال الموسـم الثـتوى لأعـوام

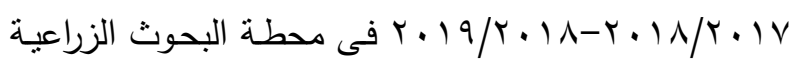
بمنطقة أبيس، والتابعـة لكليـة الزراعـة -جامعـة الأسكندرية،

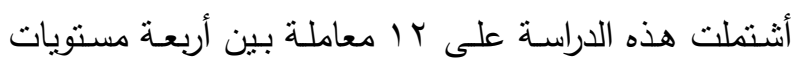

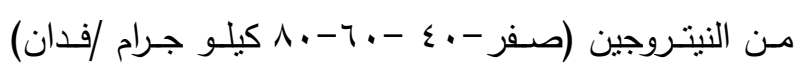
وثـلاث تركيزات من حمض الفولفيك (صفر - .. 1-...

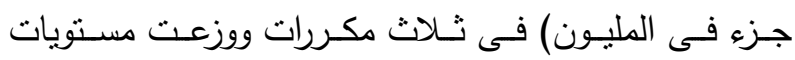

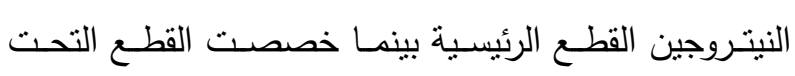
الرئيسية لحمض الفولفيك وتمت زراعة شتلات الكرنب صنف الفت

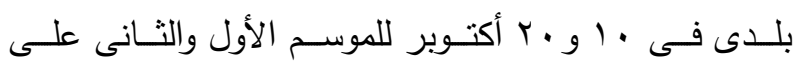
الترتيـب. وقد أظهرت النتائج أن زيـادة معدلات النيتروجين

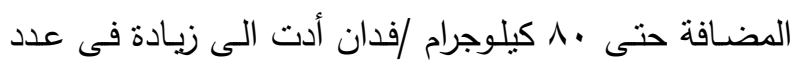

